\title{
IV.
}

\section{Das Heer Justinians}

(nach Procop und Agathias).

\section{Bestand des Heeres.}

\section{A. Die Reichsangehörigen Truppen.}

1. Die $x \alpha \tau \alpha \dot{\alpha} \circ \gamma \circ$ o.

Für die einzelnen Truppenkörper der Reichsarmee ge-

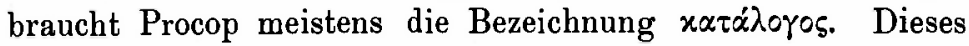
Wort bedeutet zunächst die Stanımrolle (Suid. Hes. s. v.). Da lag es denn nahe, die Reichstruppen, deren Namen in den Stammrollen verseichnet waren, als $\varepsilon x$ x $\alpha \tau \alpha \lambda \hat{\sigma}$ you zu charakterisieren (so BG II, 23 p. $238,13 \pi \varepsilon \zeta 0 i$ ह่x $x$. ; III, 39 p. 445 ,

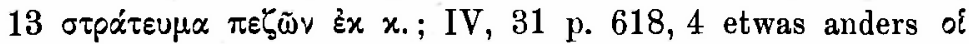
$\left.x \alpha \tau \alpha \lambda \operatorname{ó}_{\gamma} \circ \mathrm{v} \sigma \tau \rho \alpha \tau i \tilde{\omega} \tau \alpha\right)$, insofern für die nicht zum Reichsheer gehörigen Hilfstruppen verschiedener Art solche Stammrollen schwerlich existierten. Auffallend ist nur der kollektive Gebrauch des Singularis.

Mit leichter Uebertragung heiß̧t sodann der Truppenkör-

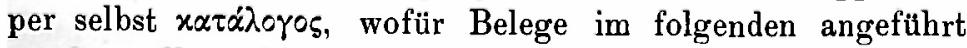
werden sollen; hier bemerken wir nur, daG sehr oft die Besatzung einer Provinz of $x \alpha \tau \dot{\alpha} \lambda$ o II, 14 p. 215,20$)$ genannt wird. Neben $x \alpha \tau \dot{\alpha} \lambda$ o 0 o gebraucht Procop aber für das Reichsheer das Wort $\sigma \tau \rho \alpha \tau i \tilde{\omega} \tau \alpha$. So spricht er BG III, 13 p. 330, 4 von einem aus $\beta \dot{\alpha} p \beta \alpha p o t$ und $\sigma \tau \rho \alpha \tau \iota \tilde{\omega} \tau \alpha \iota$ bestehenden Heere (vgl. BG IV, 31 p. $618,7 \sigma \tau \rho \alpha-$

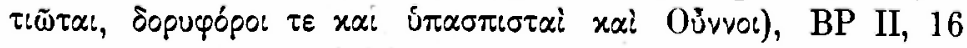
p. 324,9 von oi żv $\Lambda$ isáv $\sigma \tau \rho \alpha \tau$. (vgl. BG III, 11 p. 321, 12)

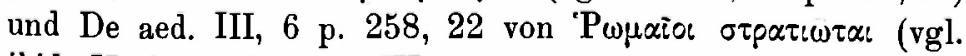
ibid. II, 9 p. 235, 13; III, 11 p. 243, 20). Es finden sich 
auch beide Bezeichnungen verbunden, wie Hist. arc. 11

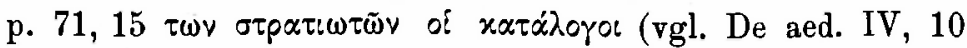

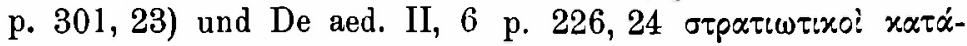

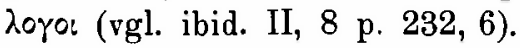

Agathias spricht nie von $x \alpha \tau \alpha$ ג.

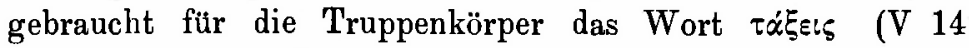

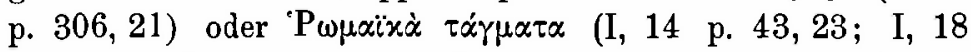
p. 52,19 ; II, 9 p. 85, 4; III, 12 p. 165, 2) oder $\sigma \tau \rho \alpha ́ \tau \varepsilon u \mu \alpha$ 'Pwuxixóv (II, 2 p. 67, 12) oder einfach 'P $\omega \mu \alpha i o r$ (II, 18 p. 180, 14).

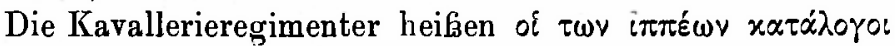

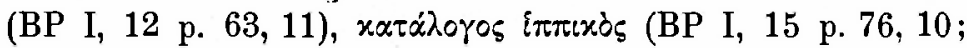
BG I, 5 p. 26, 14; I, 17 p. 86, 15 und sonst auferordentlich

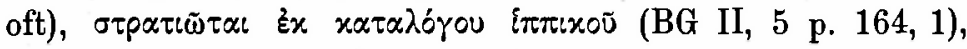

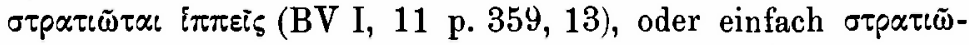

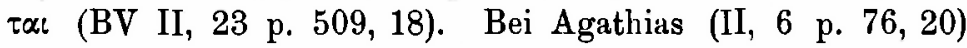
heißst eine Reiterabteilung $\tau \dot{\alpha} \gamma \mu \alpha$.

Die Infanterieregimenter heißen $\pi \varepsilon \zeta$ ol (BV II, 15 p. 481, 14;

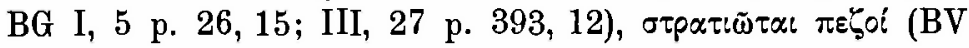

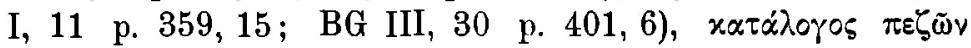

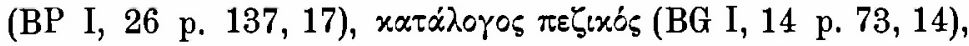

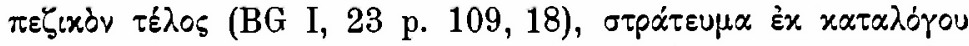

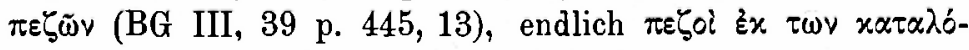

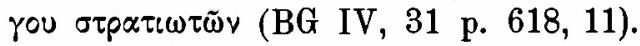

$\mathrm{Ob}$ die mit der Bedienung der Geschütze betrauten Mann-

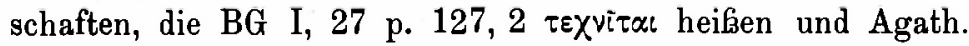
III, 25 p. 196, 4 wegen kundiger Behandlung der Maschinen gelobt werden, eigene Abteilungen gebildet haben, ist nicht zu ermitteln.

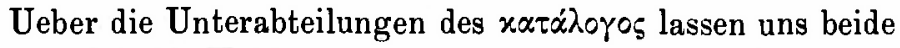
Schriftsteller im Unklaren; jedoch zerfiel das Regiment in

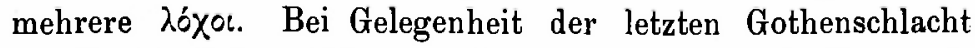
am Vesuv wird erzählt, daf die Truppen sich aufstellten, wie

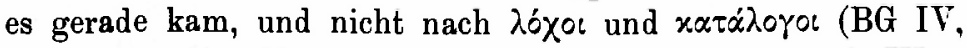
35 p. 639, 9). Uebrigens identifiziert Procop (De aed. III, 4 p. 254,8) den $\lambda o ́ x \circ \varsigma$ mit der Legion. Er spricht dort von den Vierzig Heiligen, Soldaten der leg. XII, die zu Melitene gelegen

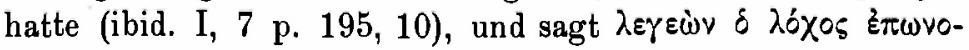


$\mu \alpha \zeta_{\zeta} \varepsilon \tau$, wobei er allerdings an ein Detachement gedacht haben kann.

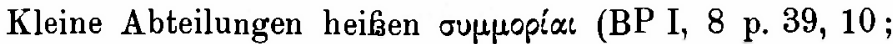
BG II, 23 p. 239,14 ; III, 5 p. 300,11 ) oder poipa (BP II, 3 p. 160,18 ; II, 18 p. 230,21 ) oder àtópospa (Ag. III, 3 p. 143,13$)$.

Die militärischen Grade anlangend, so heißst ein Gemeiner

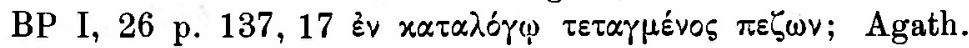

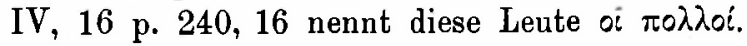

Von Prinzipalen ist nirgends die Rede außer BV II, 20 p. 448,22 , wo es von einem gewissen $\Gamma \hat{\zeta} \zeta \omega \nu$ heilit

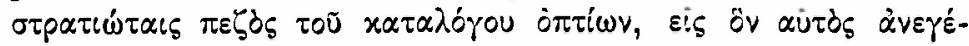

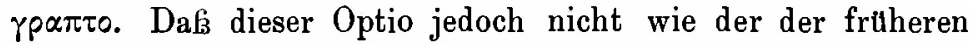
Kaiserzeit Adjutant eines Centurio war, zeigen die folgenden

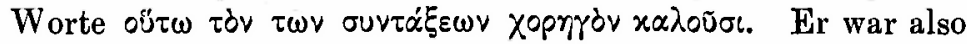

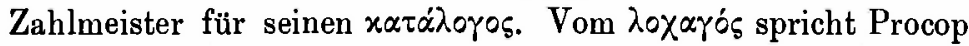
nie; dahingegen findet sich Agath. III, 21 p. 186, 11 ein

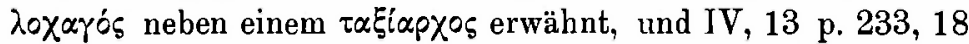
ist ein solcher mit einem wichtigen Kommando betraut. Ueberdies gab es nach demselben Schriftsteller (V, 15 p. 309, 17)

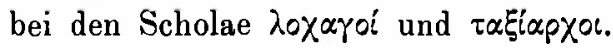

Die Kommandeure der $x \alpha \tau \alpha \dot{\lambda}$ oyo heißen bei Procop meist

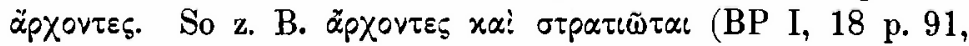

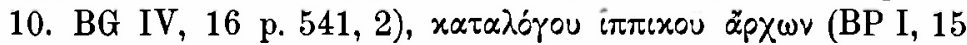

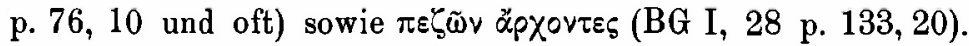
Daneben findet sich auch $\dot{\eta} \gamma \varepsilon \mu \omega \dot{\omega}$ (BV II, 15 p. 481, $13 \dot{\eta} \gamma$. $x \alpha \tau \alpha \lambda . ~ i \pi \pi$.; ibid v. $14 \hat{\gamma} \gamma \cdot x \alpha \tau \alpha \lambda . \pi \varepsilon \zeta$.). Daß auch verbale Bezeichnungen durch a้p xaเv (BG III, 38 p. 441, 17) oder

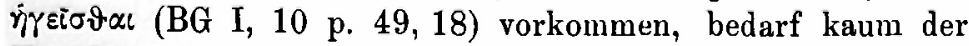
Erwähnung. Bei Agathias ist es anders; wie er die $x \alpha \tau \alpha$ -

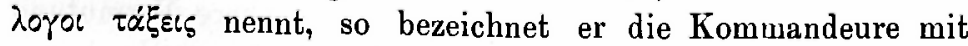

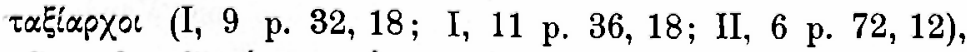

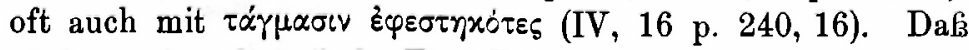
übrigens der altrömische Titel Tribunus noch in Gebrauch war, zeigen Cod. Just. I, 27, 2 und Nov. Just. 117 cap. 11.

Man sollte erwarten, daß die Kommandeure der $x \alpha \tau \alpha$ $\lambda$ ¡yo ohne Ausnahme reichsangehörige Männer waren. Dies ist aber nicht der Fall, vielmehr finden sich in dieser immer- 
hin verantwortlichen Stellung wiederholt Barbaren. So Hunnen (BG III, 23 p. 375, 5; Agath. IV, 15 p. 236, 22); Heruler (BG IV, 9 p. 498, 3 ; Agath. I, 14 p. 43, 23); ein Iberer (BG III, 28 p. 395, 14); ein Gothe (BG IV, 25 p. 593, 19); ein Gepide (BG IV, 8 p. 493, 5); ein Ante (Agath. III, 21 p. 186, 11) und zwei Barbaren unbestimmter Herkunft (BG IV, 27 p. 604, 7; Ag. III, 6 p. 150, 2).

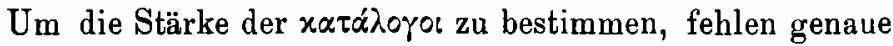
Angaben. Da sich aus den zahllosen Nachrichten über die Stärke der Truppenteile, welche in dem bunten Kriegsleben den einzelnen Offizieren zur Führung übergeben werden, Schlüsse nicht ziehen lassen, so bleiben nur einige wenige brauchbare Stellen, die aber auch nicht zu festem Ergebnis führen.

BV II, 10 p. 448 f. wird erzählt, daßs zwei Offiziere

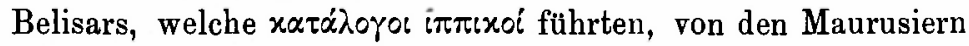
getötet wurden; nach ibid. 11, p. 454,9 wurden auch ihre Regimenter, welche zusammen 500 Mann zählten, vernichtet. Das würde für jeden $x \alpha \tau \alpha \dot{\lambda} \circ \gamma^{\prime} \circ 50$ Mann ergeben. Auf die nämliche Zahl führt BG II, 5 p. 164, 1. Hier, werden 1000 Reiter $\dot{x} x$ $x \alpha \tau \alpha \lambda \dot{c}$ you von zwei namhaft gemachten Offizieren und $\alpha \lambda \lambda \circ$ kommandiert. Nehmen wir für $\not 2 \lambda \lambda \circ$ die geringste Zahl, nämlich 2, so bekommen wir ebenfalls 250 Mann. Nach Agath. III, 6 p. 150, 1 dagegen führen 2 Offiziere, beide Komman-

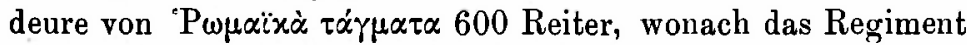
300 Mann zählen würde. Vielleicht waren jene Truppenkörper von 250 Mann nicht rollzählig.

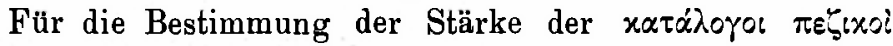
sind wir noch übler daran. Aus BG II, 23 p. 238, 13, wo von $500 \pi \varepsilon \zeta{ }_{0}$ aus dem $x \alpha \tau \alpha \dot{\lambda}$ oyos eines gewissen Demetrios die Rede ist, lernen wir nur, daß das Infanterieregiment mehr als 500 Mann zählte. Nur eine ganz unsichere Vermutung gestattet BV I, 11 p. 358, $18 \mathrm{f}$. Belisar hat zum Vandalenkriege $10000 \pi \varepsilon \zeta 0^{\prime}$ er erhalten, und zwar sind diese teils $\sigma \tau p \alpha-$ $\tau \iota \tilde{\omega} \tau \alpha \iota$, teils Foederati (über diese s. unten; daß sich bei diesen in der Tat auch Infanterie befand, zeigt BV II, 5 p. 430, 13).

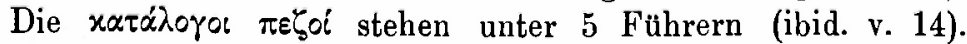
Nehmen wir nun an, daß̊ die Hälfte der Gesamtzahl, nämlich 5000 Mann, zu den Foederati gehörte, so würde jeder der ge- 


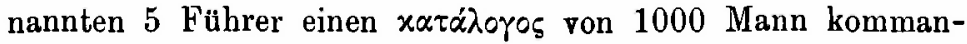
diert haben.

Die Kommandeure der von mehreren $x \alpha \tau \alpha \dot{\lambda}$ oyol besetzten Provinzen, die Duces, heißien otparryoi (BP I, 15 p. 74, 14; Agath. I, 19 p. 54,9), auch war das lateinische Wort üblich,

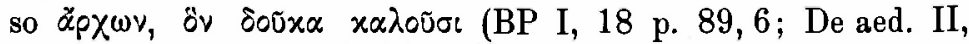
6 p. 226, 24; III, 6 p. 259, 7; vgl. III, 1 p. 248, 7), oder ein-

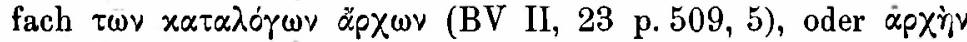

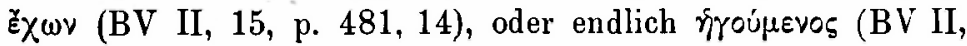
25 p. 515, 10). Solche Duces finden sich in Armenien (BP I, 15 p. 74,14 ; De aed. III, 1 p. 248,7 und 13 ; III, 3 p. 251, 15 und p. 252, 11); in Mesopotamien (BP I, 22 p. 111, 22 ; II, 14 p. 216, 6; De aed. II, 6 p. 226, 24); in Palästina (BP II, 1 p. 155, 6); in Syrien (De aed. II, 8 p. 232, 6); in Byzacium (BV II, 23 p. 509, 5; II, 27 p. 523, 9); in Numidien (BV II, 15 p. 481, 14 ; Il, 25 p. 515, 10); im Lande der Tzanni (De aed. III, 6 p. 259, 7 und 260, 14); in Moesien (Ag. I, 19 p. 54, 9). In welcher Provinz der Praefectura Illyrici der BG III, 11 p. 321, 12 erwähnte $\sigma \tau \rho \alpha \tau \iota \omega \tau \tilde{\omega} \nu \tau \omega \nu$

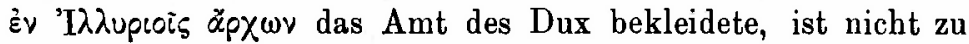
ermitteln. Wir bemerken noch, daß den Duces nach der Notitia das Prädikat spectabilis zustand.

Aus den Duces gingen die Magistri militum, viri illustres, hervor, welche auf denjenigen Punkten, wo die Verteidigung des Reiches eine einheitliche höhere Leitung verlangte, größere Truppenmassen kommandierten. Es werden deren folgende genannt.

Zunächst die Magistri praesentales mit dem Sitze in By-

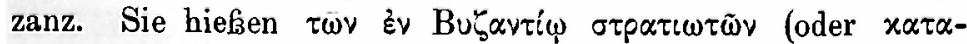

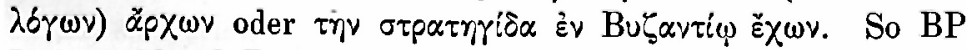
I, 8 p. 39, 16 Patricius und Hypatius, von denen der eine als Magister equitum, der andere als Magister peditum anzusehen ist; Sittas (BP I, 15 p. 74, 15), Artabanes (BG III, 31 p. 406, 19) und der Heruler Suartuas (BG IV p.593, 18).

Sodann der Magister militum Orientis (

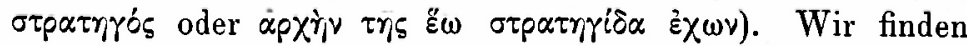
in dieser Stellung Anatolius (BP I, 2 p. 15, 2), Areobindus (BP I, 8 p. 39, 12), Justinian (BP I, 11 p. 52, 21 und I, 12 
p. 59, 11), Patricius (BP I, 11 p. 54,9), Belisar (BP I, 13 p. 61,$4 ; 21$ p. 107,$16 ; 26$ p. 137,6 ; II, 6 p. 146,13 ; IV p. 215,16 ; BV I, 11 p. 360, 20; BG IV, 21 p. 569, 8; Hist. arc. 4 p. 31, 9; Cod. Just. I, 27, 2 ; Nov. Just. 155). Seine Vertreter sind Sittas (BP I, 21 p. 107, 18), Buzes (BP II, 6 p. 176, 13) und Martinus (BP II, 24 p. 261, 16; Hist. arc. 4 p. 31,9 ).

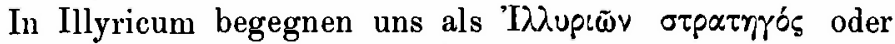

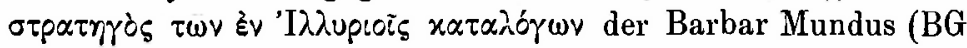
I, 5 p. 26, 4), Justinus (BG II, 13 p. 199, 9), Vitalius (BG III, 10 p. 316, 1) und Johannes, der Neffe Vitalians (BG III, 39 p. 446,8$)$.

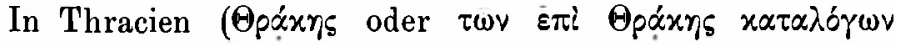

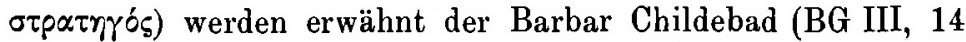
p. 331,7 ), der Arsacide Artabanes (BG III, 39 p. 445, 20) und Germanus (BG III, 40 p. 450,6 ).

Den im vorstehenden genannten, schon vorlängst eingerichteten, Kommandostellen fügte Justinian eine neue hinzu. Er setzte in Armenien einen Magister militum ('Appevíwv oder

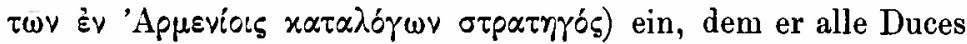
dieser Provinz unterstellte, und dessen Gewalt er auf den Pontus Polemoniacus erstreckte (C. Just. I, 29, 5; De aed. III, 1 p. 246, 21, vgl. III, 5 p. 256, 22). Procop nennt in dieser Stellung Sittas, zugleich Mag. mil. praes. (BP I, 15, p. 74, 15), Valerianus (BP II, 14 p. 215, 19; II, 24 p. 260,9) und Bessas (BG IV, 9 p. 497, 20, vgl. IV, 13 p. 525, 11).

Aus Cod. Just. I, 27, 2 erhellt, daß in dem wiedergewonnenen Afrika ein Mag. mil. nicht eingesetzt wurde. Die militärischen Oberbefehlshaber sind jedoch Belisar (BV, I, 11 p. 360,20 ), Solomon (BV II, 8 p. 444, 14), Germanus (II, 16 p. 482, 19), Solomon (II, 19 p. 493, 4), Sergius II, 22 p. 506, 1), Sergius und Areobindus (II, 24 p. 513, 15), Areobindus (II, 24 p. 515, 8), Artabanes (II, 28 p. 533, 4) und Johannes, Bruder des Pappus (II, 28 p. 533, 7).

In Italien erscheint nur Demetrius als Mag. mil. (BG III, 6$, p. 303,1$)$.

In Afrika wird zweimal ein Magister peditum erwähnt,

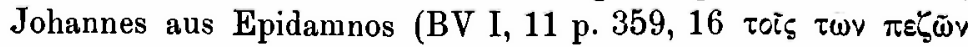




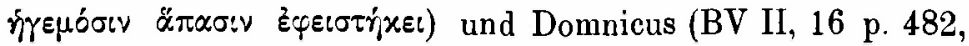

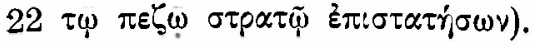

Die höchste militärische Stellung war die des unumschränk-

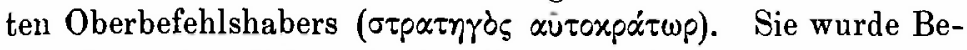
lisar dreimal verliehen, für den Vandalenkrieg (BV I, 11 p. 360, 20) mit dem Zusatz, daf seine Anordnungen als kaiserliche gelten sollten (p. 361, 1), und zweimal für den Gothenkrieg (BG I, 5 p. 26, 18; II, 22 p. 235, 5), ferner dem Germanus (BG III, 39 p. 446, 3), dem Narses (BG IV, 21 p. 570,3) und endlich dem Justinus im Colcherlande (Agath. IV, 21 p. 250, 21).

Auffallenderweise wurden mitunter hochgestellte Zivilbeamte mit militärischen Funktionen betraut. So unter Anastasius der Magister officiorum Celer im Perserkriege (BP I, 8 p. 39,14$)$, und dem Belisar wurde Hermogenes, der dasselbe

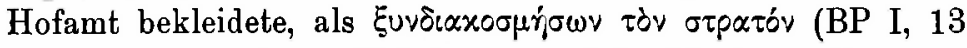
p. 61, 7) beigegeben. Uebrigens stand der Magister officiorum, da er die Scholae kommandierte, dem Militär nicht ganz fern. Merkwürdiger ist, daß Narses, der als Comes sacrarum largitionum ein reines Zivilamt bekleidete, als General und später als Generalissimus nach Italien geschickt wurde (BG II, 13 p. 199,4$)^{1}$ ); IV, 21 p. 570,3 ), und daf Justinian, als in Italien die Sache der Römer sehr schlecht stand, einen Praefectus praetorio für Italien ernannte und diesen den hohen Offizieren vorsetzte (BG III, 6 p. 302, 13).

\section{Reichsangehörige Sondertruppen.}

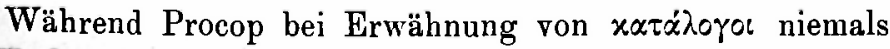
die Herkunft der betreffenden Mannschaften bezeichnet, nennt er mehrfach $n$ eben den $x_{\alpha} \alpha \alpha \dot{\lambda}$ oyor, also nicht zu ihnen gehörig, aus Reichsangehörigen gebildete Truppenkörper mit ihrem Stammesnamen. Die hier in Betracht kommenden Stämme

1) Der Umstand, daßs Narses Eunuch war, bildete bei den ausgezeichneten Eigenschaften dieses Mannes kein Hindernis. Den Wechsel der Anschauungen zeigt Dig. 49, 16,4, wonach Traían besonders verfügt hatte, daf Leute, die nur mit einem Testikel geboren waren, oder einen Testikel verloren hatten, im Heere dienen durften. Ein Eunuch wäre also damals nicht zugelassen. Auch Solomon, der im Vandalenkriege sich auszeichnete, war durch einen Unglücksfall in seiner Kindheit Eunuch geworden (BP I, 11 p 359,8). 
sind die halbbarbarischen Bergvölker Thraciens und Illyriens, sowie die niemals vollständig unterworfenen Isaurier. Es ist übrigens nicht anzunehmen, daß aus diesen Stämmen nie ein

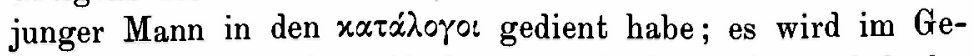
genteil Hist. arc. 6 p. 43,2 ausdrücklich bezeugt, daf der spätere Kaiser Justinus mit zwei Genossen, alle drei Illyrier, arme Ackerknechte, nach Byzanz gewandert und dort in die $x \propto \tau \alpha \dot{\lambda}$ oyou eingetreten sei.

Unsere Kenntnis dieser nationalen Truppenkörper ist sehr beschränkt; oftmals ist nicht $z u$ erkennen, ob es sich um Kavallerie oder Infanterie handelt. Im Einzelnen ist folgendes zu bemerken. BP II, 21 p. 244,3 werden in Belisars Heere Thraker und Illyrier mit Gothen, Herulern, Vandalen und Maurusiern koordiniert. BG II, 5 p. 163, 23 werden 800 thracische Reiter unter einem nationalrömischen Führer neben

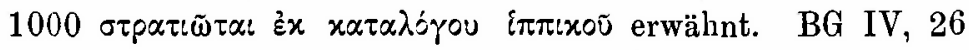

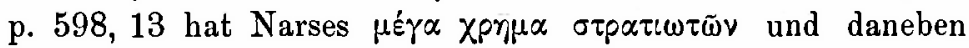

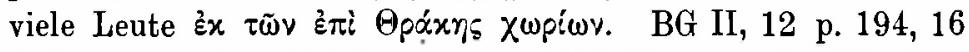
finden wir eine Abteilung von 1000 Mann, teils Thraker, teils Isaurier, jeder Stamm mit einem besonderen Führer, wie es scheint, gleicher Nationalität. BG III, 6 p. 302,16 werden Thraker zur See nach Italien geschickt. Wir dürfen unbedenklich annehmen, dafs diese Mannschaften angeworben wurden. BG IJI, 10 p. 315,24 werben Belisar und Vitalius in Thracien 4000 Mann an (vgl. BG III, 12 p. 325,5), und BG III, 39 p. 446,5 beauftragt Justinian den Germanus, in Thracien Truppen zu werben (vgl. ibid. p. 447, 7). Hier möge noch bemerkt werden, daß Thracier mehrfach als Offiziere bei

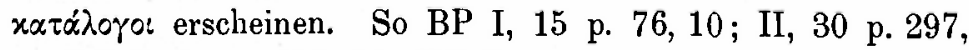
3 ; BV I, 11 p. 359,21 ; BG I, 5 p. 26, 11.

Seltener werden Illyrier erwähnt. BP II 21 p. 244,3 finden sie sich in Belisirs Heere; BG III, 10 p. 316,3 und 320,15 werden 'I $\lambda \lambda$ upıoi $\sigma \tau p \alpha \tau i \omega \tilde{\tau} \alpha \iota$ genannt; p. 320,19 ziehen diese eigenmächtig ab, weil sie lange Zeit ihre Kompetenzen nicht bekommen haben. BG IV, 26 p. 598, 13 hat Narses

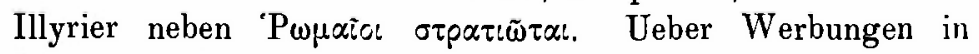
Illyrien s. BG III, 12 p. 325,5 und III, 39 p. 446, 5 .

Isaurier werden neben Reichstruppen öfters genaunt. BP 


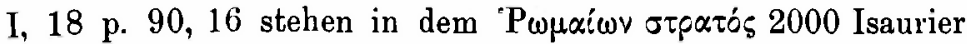
unter 2 Fuhrern. BG I, 10 p. 49, 18 erscheinen sie neben

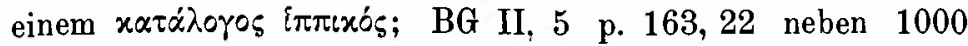

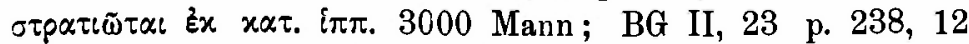

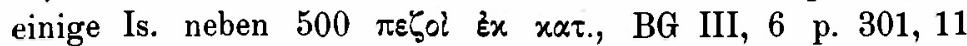
1000 Mann teils 'P $\omega \mu \alpha i o c$, teils Isaurier. Auch sonst ist mehrfach von ihnen die Rede. BP I, 18 p. 95, 22 werden junge Isaurier direkt vom Pfluge her ohne alle militärische Ausbildung eingestellt; es wird aber ihre Kampfbegier gerühmt (ibid. p. 96, 4). BG I, 5 p. 26,10 finden sich 3000 Is.; BG II, 12 p. 194, 161000 Mann teils Is., teils Thracier. BG II, 27 p. 256, 9 (vgl. p. 258,8) verstehen sie sich auf Maurerarbeiten; BG III, 10 p. 318,13 bilden sie die Besatzung von Tibur; BG III, 20 p. 360,21 verraten sie zum ersten und III, 36 p. 433, 8 zum zweiten Male Rom. Uebrigens dienten unter ihnen auch Lycaonier (BP I, 18 p. 96, 6).

Einige Male werden auch kleine Abteilungen von Armeniern erwähnt, die fast stets von Landsleuten kommandiert werden. So BP II, 21 p. 243, 12; BG III, 26 p. 390, 17; IV, 11 p. 516, 5. Eine Ausnahme findet sich BG III, 6 p. 302, 16, wo eine größere Schar Armenier unter einem iberischen Offizier steht.

\section{U e b e r l ä u f e r.}

Im römischen Heere dienten auch Ueberläufer. So $B G$

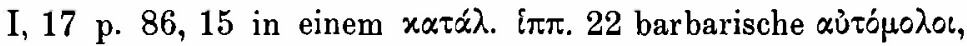
deren Herkunft nicht genannt wird. BG IV, 26 p. 599, 1 führt ein persischer Prinz, namens Cabades, unter Narses viele Perser. Mehrfach ist von vornehmen Armeniern aus dem den Persern gehörenden Teile des Landes die Rede, die zu den Römern übertreten und von diesen mit Kommandostellen bekleidet werden. Vor allen ist der große Narses Persarmenier (BP I, 15 p. 79,7). Ein gewisser Varazes führt einmal 800

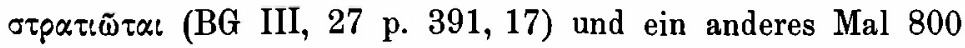
Tzanni (BG IV, 13 p. 525, 10). Ferner gehören hieher zwei Brüder, Aratius und Narses, die im Jahre 531 mit ihrer Mutter nach Byzanz ziehen (BP I, 15 p. 79, 3) und von denen der erstere zu Belisar nach Italien geht (BG II, 13 p. 199, 11), 
der letztere unter Narses dient (vgl. ibid. v. 10), als dieser zum ersten Mal nach Italien geschickt wird. Beide Brüder futhren Armenier (BG II, 27 p. 257, 19). Eine bedeutende Rolle spielte der persarmenische Ueberläufer Artabanes, ein Arsacide (BG IV, 8 p. 494, 5). Ursprünglich den Römern feindlich, wie er denn den Sittas tötete (BP II, 3 p. 162, 4), trat er später zu den Römern mit einigen Armeniern über (BV II, 24 p. 513, 10) und wirkte hervorragend im Vandalenkriege (BV II, 25 p. 515,$21 ; 27$ p. 523,$18 ; 28$ p. 527, 14). Daß er eine Zeitlang den Oberbefehl in Afrika fuhrte, ist bereits oben S. 106 erwähnt.

\section{Kriegsgef angene.}

Nicht selten traten Kriegsgefangene, zum Teil um einem schlimmeren Geschick zu entgehen, in das römische Heer ein. Pelisar erorberte die persische Festung Sisauranon und sandte die Besatzung nach Byzanz; Justinian schickte diese Leute später nach Italien, wo sie von einem Armenier geführt wurden (BP II, 19 p. 235, 20; BG III, 3 p. 291, 15). Nach BV II, 14 p. 471, 12 wurden vandalische Gefangene zunächst nach Byzanz geschafft und von da in den Orient geschickt, um unter

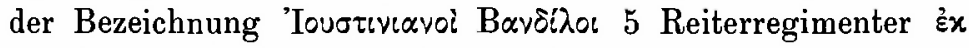
$x \alpha \tau \alpha \lambda$. zu komplettieren. Etwa 400 von ihnen entkamen jedoch auf der Seefahrt und flüchteten nach Afrika. Vandalen finden sich ferner in Belisars Heere (BP II, 21 p. 244, 5), und solche bildeten einen Teil des glänzenden Gefolges, mit dem Belisar in Byzanz aufzog (BG III, 1 p. 281, 3). Was die Gothen anlangt, so dienten die mit Vitigis nach Byzanz gebrachten Leute im Orient (BP II 14 p. 216, 2; 18 p. 232, 4; 21 p. 244, 4). BG IV, 27 p. 260, 14 treten nach der Einnahme von Auximum die gefangenen Gothen in das römische Heer. BG II, 29 p. 272, 2 unterwerfen sich Belisar mehrere Gothenführer und bleiben bei ihm. BG III, 12 p. 329,9 befehligt der den Römern freundlich gesinnte Sisifrid die römische Besatzung von Assisi. BG IV, 25 p. 593, 19 wird Amalafrid, ein vornehmer Gothe aus königlichem Geschlechte, der mit Vitigis nach Byzanz gekommen war, vom Kaiser zum Offizier ( $\left.\not \alpha_{\rho} \chi \omega v\right)$ ernannt und mit einem Kommando betraut. 
Selbstverständlich befanden sich auch viele Gothen in dem erwähnten Gefolge Belisars (BG III, 1 p. 281, 3).

\section{B. Die barbarischen Hilfstruppen.}

Ein sehr wesentliches Element des römischen Heeres bildeten die von barbarischen Grenznachbaren auf Grund von Verträgen gegen Zahlung bedeutender Summen gestellten Hilfsvölker, von denen folgende genannt werden.

1. Die Hunnen. Von hunnischen Stämmen erwähnt Procop die Kuturguren, Uturguren und Sabiri, meist spricht er einfach von Hunnen. Die Kuturguren (BG IV, 18 p. 552,3) wohnten westlich, die Uturguren (ibid. v. 17) östlich von der Palus Maeotis, die Sabiri am Kaukasus (BP II, 29 p. 288, 22; BG IV, 3 p. 469, 22). Geldzahlungen an die Hunnen werden erwähnt BP II, 10 p. 198, 13; BG III, 12 p. 326, 3; Hist. arc. 8 p. 53, 20, insonderheit jährliche Zahlungen und Geschenke an die Uturguren BG IV, 18 p. 553, 4. 11. Die unter sich nicht einigen Sabiri erhielten sogar von den Römern und Persern Geld; daher finden sie sich auch im Heere der letzteren (BG IV, 11 p. 509, 19; 13 p. 524, 17; 14 p. 529, 20); auf Seiten der Römer stehen sie Agath. III, 17 p. 177, 1. Ohne nähere Bezeichnung des Stammes begegnen uns die Hunnen oft als Bundesgenossen der Römer, z. B. BP II, 26 p. 270,19 ; BV I, 17 p. 381,20 ; BG I, 27 p. 125,20 und 129, 8. Sie sind gute Bogenschützen (BG II, 1 p. 146, 2), namentlich i $\pi \pi \circ \tau \circ \xi \hat{o} \tau \alpha:$ (BG I, 27 p. 129, 8). Uebrigens sind sie nicht zuverlässig. Sie stellen sich, um je nach dem Ausgange der Schlacht freie Hand zu behalten, niemals bei den Römern, sondern stets getrennt auf (BV II, 3 p. 420, 23), versprechen sogar den Vandalen, eventuell zu ihnen überzutreten (BV II, 1 p. 412, 11). Auch verzichteten sie keineswegs auf räuberische Einfälle in römisches Gebiet (BG IV, 21 p. 572, 1, Hist. arc. 18 p. 108, 13); besonders bedrohlich war ein Zug der Kuturguren gegen Byzanz (Ag. V, 12 p. 302, 15). Höchst auffallenderweise nennt Procop nicht selten die Hunnen mit dem Namen Massageten, an deren Stelle jene getreten waren. So BV I, 4 p. 329, 7, wo die Scharen Attilas Massageten heißen; I, 11 p. 359,21 vgl. mit p. 360,3 ; I, 18 
p. 386,11 und 12 ; BG II, 1 p. 145,7 vgl. mit 146, 2 ; Hist. arc. 7 p. 48,6. Es ist daher anzunehmen, dafo auch an denjenigen Stellen, an denen die Identifizierung der beiden Namen nicht nachzuweisen und einfach von Massageten die Rede ist (BV I, 12 p. 364,2 ; I, 17 p. 381, 20; BG I, 10 p. 53, 21), Hunnen gemeint sind ${ }^{2}$ ).

2. Ein Teil der Heruler wohnte zu Justinians Zeit in den ihnen von diesem angewiesenen Sitzen um Singidunum (BG III, 23 p. 419, 1). Sie bezogen Lieferungen (BG III, 33

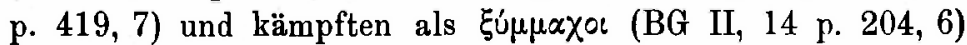
stets auf Seiten der Römer (BP I, 13 p. 62, 15; BV I, 11 p. 360,1 ; BG II, 4 p. 160,10 ; Agath. I, 11 p. 36,13 und sonst sehr oft). Das hinderte sie jedoch nicht, Raubzüge in Illyrien und Thracien zu unternehmen (BG III, 33 p. 419,2). Auch sonst waren sie nicht völlig zuverlässig. BG II, 22 p. 235, 8 wenden sie sich für kurze Zeit den Gothen zu, und Ag. II, 7 p. 78,12 ist von einer Mißstimmung gegen die Römer die Rede.

3. Ein nur kleiner Teil der Ostgothen war nicht mit Theoderich nach Italien gezogen, sondern in den alten Wohnsitzen am Cimmerischen Bosporus geblieben. Sie hieß̧en Гórło

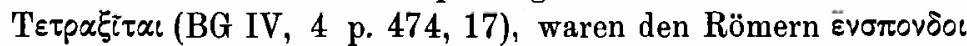
und leisteten auf Wunsch des Ǩaisers Zuzug (De aed. III, 7 p. 262, 8).

4. Die Gepiden wohnten um Sirmium und Singidunum auf beiden Ufern der Donau (BV I, 2 p. 313, 11; BG I, 3 p. 19,2 ; III, 33 p. 418,3 ; H. arc. 18 p. 108, 8). Sie schlossen ein Bündnis mit Justinian (BG IV, 25 p. 593, 1); 400 von ihnen dienten in Narses' Heere (BG IV, 26 p. 599, 6); ein Gepide versetzte Totilas den Todesstof (BG IV, 32 p. 624, 21 ff.).

5. Den Lan g obard en gab Justinian aufer großen Geldsummen Wohnsitze in Pannonien (BG III, 33 p. 418, 11) und schloß mit ihnen einen Vertrag (BG III, 34 p. 426, 3; IV, 25 p. 593,9 und 594,14$)$; sie werden daher als $\varepsilon v \sigma \pi o v \delta o$ bezeichnet (BG III, 33 p. 418, 17). Wir finden sie im Heere

2) Bei Ammianus 22, 8, 38; 23, 5, 16; 31, 2, 12 werden die Massageten mit den Alanen identifiziert. 
des Germanus (BG III, 39 p. 447,20) und des Narses (BG IV, 26 p. 598, 18), der sie jedoch wegen ihrer ungebändigten Wildheit, allerdings reich beschenkt, fortschickt (BG IV, 33 p. 627,19$)$.

6. Von den V arni, die nach Procops (BG IV, 20 p. 559, 3) unklarer Angabe bis zum Rhein und zur Nordsee wohnten, wird nur erzählt, daß3 sie von Narses große Geschenke erhielten und mit diesem zu Ariminum ein Bündnis abschlossen (Ag. I, 21 p. 59, 8).

7. Die Saracenen wohnten an der Südostgrenze des Reiches rom Euphrat bis zum arabischen Meerbusen (BP I, 17, p. 88, 5). Der eine Teil von ihnen stand unter persischer: Hoheit (ibid. p. 89, 3), der andere war mit den Römern ver-

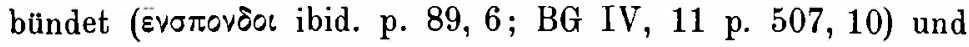
erhielt jährliche Zahlungen (BP II, 10 p. 198, 13). So erscheinen sie denn auch auf Seiten der Römer (BP I, 17 p. 89,$6 ; 18$ p. 90,18$)$.

8. und 9. Die Antae und die Sclaveni, slavische Völkerschaften, waren den Römern feindlich. Erstere wohnten jenseit der Donau (BG I, 27 p. 125, 19; III, 14 p. 336, 5), nördlich von den Uturguren (BG IV, 11 p. 474, 14), letztere waren ihre westlichen Nachbaren (BG III, 40 p. 450, 4). Beide Völkerschaften machten häufig Einfalle in Illyrien und Thracien. Ueber solche der Sclaveni s. BG III, 13 p. 330 , 15 ; 14 p. 331,12 ; 29 p. 397,18 ; Hist. arc. 18 p. 108, 11 und sonst. Betreffend die Antae s. BG III, 13 p. 331, 12; Hist. arc. 18 p. 108, 14. Indessen erscheinen, wahrscheinlich als geworbene Leute (BG I, 27 p. 125, 20), sclavenische Reiter im römischen Heere. Auch Antae dienten gegen die Gothen (ibid. und BG III, 22 p. 370, 7; p. 373, 16).

Die im folgenden genannten Völkerschaften waren von den Römern abhängig.

10. Die Tzanni wohnten auf römischen Gebiete um

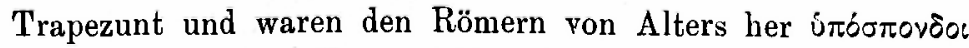
und xaríx p. 164, 11). Früher zahlte man ihnen, um ihre Einfalle abzukaufen, jährlich Geld (BP I, 15 p. 77, 17). Justinian unter-

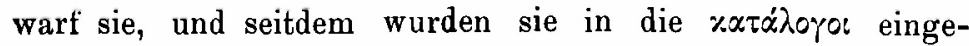


schrieben und zogen mit den Römern zu Felde (BP I, 15 p. 78,7). Wir finden sie auf Seiten derselben BP II, 29 p. 288, 4; BG IV, 13 p. 525,10 ; Ag. HI, 20 p. 184, 22. Nach einer auf einem Raubzuge erlittenen Niederlage wird ihnen ein jährlicher Tribut auferlegt (Ag. V, 2 p. 281, 5).

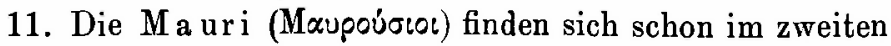
Perserkriege im Heere Belisars (BP II, 21 p. 244, 5) und später im Gothenkriege (BG I, 5 p. 26, 18; 29, p. 137, 17). Es sind treffliche leichte Truppen (BG I, 25 p. 120, 11); $\pi \varepsilon \lambda \tau \alpha-$

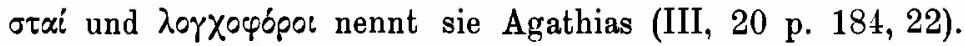

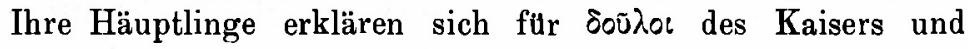
empfangen von ihm ihre Insignien, aber auch Geld (BV I, 25 p. 406,15$)$.

\section{Die Privatsoldaten.}

Außer den genannten Truppenteilen gab es zwei Arten von Privatsoldaten, die Foederati und die sopuçbor $x \alpha i$ ' $\mathrm{r} \pi \alpha \sigma \pi \iota \sigma \tau \alpha i$.

1. Mit den Foederati hatte es folgende Bewandtnis. Procop sagt BV I, 11 p. 358, 20, in früheren Zeiten seien Foederati lediglich auswärtige Völker genannt, welche nicht als Untertanen, sondern, weil sie nicht von den Römern besiegt wären, auf gleichem Fuße sich an den Staat angeschlossen hätten; allein gegenwärtig könnten ohne Bedenken Leute aus allen Völkerschaften sich diesen Namen anmaßen. $\mathrm{Zu}$ den Foederati älterer Form gehörten nun die im vorstehenden als

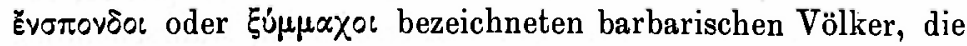

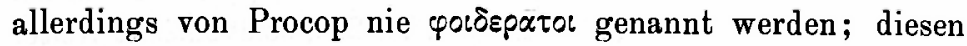
Namen spart er für die Fòderati jüngerer Form auf. Es waren dies Truppenkörper - vorwiegend Reiter; doch finden sich auch Infanteristen (BV II, 5 p. 430, 13) -, die von unternehmungslustigen Condottieri angeworben und dem Staate als wertvolles Truppenmaterial zugeführt wurden. Eingehender spricht darüber Procop BV I, 11 p. 358, 19 und 359, 5, wo 9 derartige Abteilungen unter besonderen Führern erwähnt werden. Im Vandalenkriege ist oft von ihnen die Rede (s. I, 19 p. 388,20 ; II, 3 p. 420,$16 ; 7$ p. 439,$23 ; 15$ p. 481 , 12); in den Büchern über die Perserkriege kommen sie nicht 
vor, woraus indessen nicht zu schließen ist, daß solche Truppen an demselben nicht teilgenommen hätten. Im Gothenkriege werden zwar keine Führer erwähnt, wohl aber BG I,

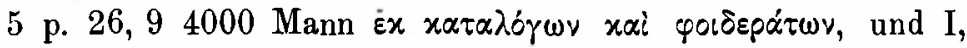
27, p. 125, 21 werden Hunnen, Sclaveni und Antae, sowie III, 33 p. 419, 4 Heruler als Foederati genannt. Daß auch Gothen sich unter ihnen befanden, zeigt Cod. Just. I, 5, 12. Wir dürfen auch unbedenklich annehmen, dafi da, wo Truppen

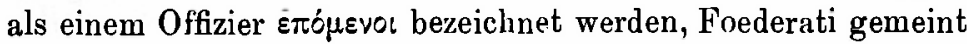
sind, z. B. BP II, 24 p. 262, 1 (Heruler), BV II, 13 p. 463, 4 (Hunnen), BG II, 5 p. 163,24 vgl. mit II, 7 p. 176,10 (Thraker). Fast alle Foederati waren Barbaren, weshalb auch von ihnen orthodoxes Bekenntnis nicht gefordert wurde (C. Just. I, 5, 12). Die Stärke der Corps war sehr verschieden. BG I, 27 p. 125, 20 haben zwei Führer 1600, BV II, 13 p. 463, 5 hat ein Führer 70 Reiter. Die Kosten für die Foederati trug der Staat. Ursprünglich werden die Kompetenzen an den Führer gezahlt sein. Als Vitalianus sich gegen

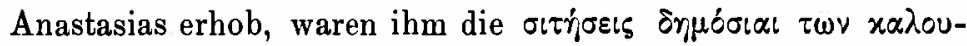
$\mu \dot{v}^{\prime} \omega \nu \vee \varphi \circ \delta \varepsilon p \alpha \tau i x \omega \vee \dot{\alpha} \vee v \omega v \tilde{\omega} \vee$ vorenthalten (Joh. Antioch. Hermes VI, p. 344, 7). Später zog man vor, kaiserliche Beamte mit der Verteilung zu beauftragen. So finden sich Chartularii fortissimorum numerorum vel foederatorum (Cod. Just. 12, 37 $(38)$; 19, 2) und ein optio genannter Offizier, der fast die Stelle eines Kommandeurs eingenommen zu haben scheint (Cod. Just. 4, 65, 35, Nov. Just. 116, 1; 117, 11). Die Foederati gingen also mehr in die Hand des Kaisers über. Was mit den Leuten geschah, wenn ihr Führer gestorben war oder eine höhere Stelle erhalten hatte, ist nicht überliefert. Wir dürfen vermuten, daß sich die Truppe entweder auflöste, oder vererbt wurde. Auf letzteres führt $B G$ II, 7 p. 176, 22, wo der Neffe des Valerianus, der BV I, 11 unter den Führern der Foederati genannt wird, inzwischen aber Magister militum in Armenien geworden ist (BP II, 14 p. 215, 18), von den $B \alpha-$

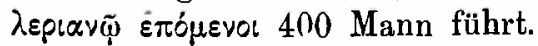

Die ganze Institution brachte nicht nur dem Staate, sondern auch den Condottieri großen Nutzen. Jener erhielt Truppen, die besser waren als die Reichsarmee. So nennt sie 
denn auch Procop BV I, 11 p. 359, 6 ff.; II, 15 p. 481, 12

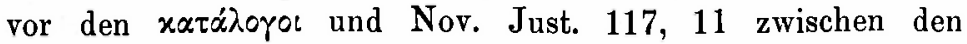
Scholae und den Numeri. Die Führer machten ein gutes Geschäft, da ihnen durch die sogen. stellatura, über die ich Philol. LXV p. 305 gehandelt habe, gewisse Teile der den Soldaten zustehenden annona zufielen. Auferdem hatten sie die beste Aussicht auf hole Kommandostellen. So wurden von den BV I, 11 p. 359 genannten 9 Führern zwei Magistri militum, und zwar Valerianus, wie schon erwähnt, in Armenien, Martinus im Orient (BP II, 24 p. 216, 6); Solomon erhielt das Kommando in Afrika (BV II, 19 p. 493, 4), und Marcellus wurde Dux Numidiae (BV II, 15 p. 481, 15). Auch der Kombination eines hohen militärischen Amtes mit der Führerschaft der Foederati stand nichts im Wege. Nach BG III, 31 p. 406, 19 war Artabanes in Byzanz Magister militum

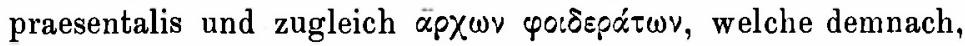
vermutlich weil die Scholae im Nikaaufstande völlig versagt hatten, auch nach der Reichshauptstadt verlegt worden wareu.

2. Ueber die zweite Klasse von Privatsoldaten haben wir folgendes zu bemerken. Wir kennen aus Tacitus' Germania 13 das Institut der Gefolgschaften. Dieses hatte auch bei den Römern Eingang gefunden. Nach kleinen Anfängen (Claudian. in Rufin. II, 76) war es zu Justinians Zeit durchaus üblich,

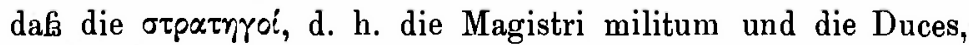
ihre Gefolge hatten. Procop nennt diese Privatsoldaten an

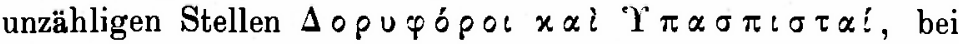
Agathias (I, 15 p. 44,20 vgl. mit p. 45,7 ) heißen sie $2 \pi \alpha-$

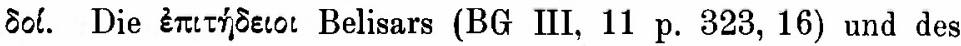
Narses (BG IV, 29 p. 610,14) werden zu ihrer Zahl gehört

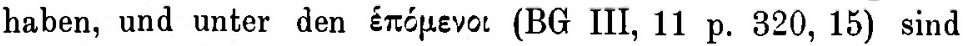
wohl $i \pi \alpha \sigma \pi \iota \sigma \tau \alpha i$ Belisars zu verstehen. Findet sich das Gefolge bei Zivilbeamten, so ist das Ausnahme. Eine solche bilden Rusticus (Agath. III, 2 p. 140, 4 vgl. mit III, 4 p. 145, 3) und ein Praefectus praetorio, der deswegen ausdrücklich notiert wird (BP I, 25 p. 130, 20). Die Zahl der Gefolgsleute war mitunter sehr grof. Belisar, der sehr reich war (BG III, 1 p. 282, 19), hatte deren 7000 Mann (ibid. p. 283, 2). Dem Valerianus, Mag. mil. in Armenien, folgten mehr 
als 1000 (BG III, 27 p. 391, 20), dem Narses dagegen weit weniger (Ag. I, 19 p. 55, 19). Die Kosten der Anwerbung und Unterhaltung des Gefolges trug der Gefolgsherr. Agathias (IV, 22 p. 252) erzählt von einem geriebenen und gewissenlosen Geschäftsmanne, der von Justinus, dem Kommandanten im Kolcherlande, ein Kapital entleiht, dieses durch unerhörte Bedrückung der Bevölkerung auferordentlich vermehrt und schließlich dem Justinus das Kapital zurückzahlt sowie demselben sämtliche durch sein Gefolge und sein Hauswesen verursachten Kosten zur Verfügung stellt. Diese Erzählung hätte keinen Sinn, wenn das Gefolge vom Kaiser bezahlt wäre. Belisar soll sich erboten haben, den zweiten italienischen Krieg auf eigene Kosten, natürlich mit seinem starken Gefolge, zu führen (Hist. arc. 4 p. 35, 7). Derselbe hatte einen eigenen Zahlmeister (optio) für sein Gefolge (BP I, 17 p. 381, 14).

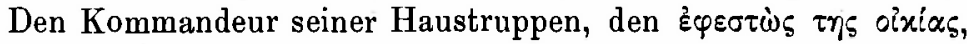
ernannte der Gefolgsherr selbst (BG II, 28 p. 261, 16; III, 36 p. 434, 16; Agath. II, 8 p. 80,4). Die Mannschaften wurden zunächst für den Gefolgsherrn, aber zugleich auch für den Kaiser in Eid und Pflicht genommen (BV II, 18 p. 491, 5). Es stand ihnen übrigens frei, zu einem andern Herrn überzutreten. So wird ein Sopuéópos des Cappadociers Theodorus von Germanus, dem Neffen Justinians, übernommen (BV II 18 p. 491, 9), zu dem auch andre Gefolgsleute unter Verlassung ihrer Herren übertreten (BG III, 39 p. 447, 12). Doch sind das Ausnahmen; im allgemeinen war das Verhältnis der beiden Faktoren ein so festes, daß man die Gefolgsleute geradezu als Eigentum des Herrn ansah, wie denn Justinian, als Belisar in Ungnade gefallen war, dessen Gefolge an seine Günstlinge verschenkte (Hist. arc. 4 p. 31, 10). Nach Ableben des Führers scheint das Gefolge unter Umständen durch Erbschaft auf einen andern Herrn übergegangen zu sein, wie Johannes, der Neffe Vitalians, neben seinem eigenen Gefolge das von seinem Schwiegervater Germanus hinterlassene dem Narses zuführt (BG IV, 26 p. 598, 14).

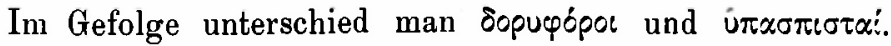
Die ersteren hatten die Stellung von Kavallerieoffizieren. Ein solcher wurde den Persern als Geisel gegeben (BP I, 22 p. 111, 
8), und Gelimer legte grołen Wert darauf, einen Sopuৎópos gefangen zu nehmen (BV I, 23 p. 402, 4). Die letzteren waren einfache Kavalleristen, fanden sich aber wohl nur bei den höchsten Offizieren. Sie wurden höher geachtet als die Katalogsoldaten; ein solcher wird von Narses zur Belohnung für bewiesene 'Tapferkeit unter seine $\dot{\pi} \pi \alpha \sigma \pi \iota \sigma \tau \alpha$ ! aufgenommen

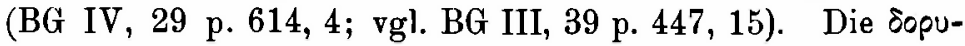
५ópor dagegen bildeten stets die unmittelbare Umgebung des Herrn. Bei Festmahlzeiten standen sie hinter dem Platze desselben, mußten aber die ihnen gereichten Speisen außerhalb des Speisesaales verzehren (BV II, 28 p. 528, 4; vgl. II, 18 p. 491, 18). Im Felde lagerten sie neben dem Herrn (BG II, 23 p. 239, 19); in der Schlacht umgaben sie ihn (BV II, 3

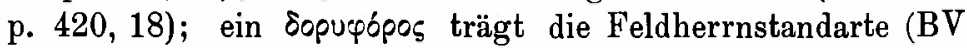
II, 2 p. 415,20 ; II, 3 p. 420,20 ; 421, 20; II, 10 p. 448, 4). Mit großer Treue schützen sie des Herrn Leben. Germanus und Solomon, die von ihren Pferden abgeworfen sind, werden durch sie gerettet (BV II, 17 p. 498,8 ; II, 21 p. 505, 16). Mit den Schilden halten sie die Belisar bedrohenden Pfeile ab (BG I, 18 p. 88, 18); ja, ein Eopuৎópos fängt mit der Hand ein auf Belisar zufliegendes Geschoß auf (BG II, 27 p. 257, 12). Die Nationalität der Gefolgsleute ist nur in verhältnismäßig wenigen Fällen angegeben; wir finden jedoch Cappadocier (BG I, 29 p. 137, 14), Cilicier (BG III, 36 p. 434, 15), Hunnen (Massageten) (BP II, 26 p. 270, 21; BV II, 10 p. 447, 22 ; BG I, 16 p. 81,4 ; II, 1 p. 148,5 ; II, 2 p. 150,20 ; II, 13 p. 198, 22; III, 30 p. 402, 6), Isaurier (BG I, 9 p. 47, 14; I, 29 p. 137,12 ; II, 10 p. 187, 8), Perser (BG II, 2 p. 150,20 ; III, 11 p. 324, 5), Pisidier (BG I, 28 p. 133, 8) und Thracier (BV II, 10 p. 447, 22; BG II, 2 p. 150, 21; II, 13 p. 198, 22 ; III, 11 p. 324, 6). Belisars Zahlmeister (optio) ist Armenier (BV I, 17 p. 381, 15). Selbstverständlich stand dem Herrn die Strafgewalt über das Gefolge zu.

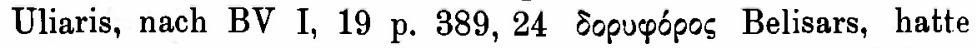
Mailand keine Hilfe gebracht und wurde deshalb von Belisar aus seiner Nähe verbannt (BG II, 22 p. 235, 2). Germanus ließ den Maximinus wegen hochverräterischer Umtriebe aufhängen (BV II, 18 p. 492, 23). Außerordentlich oft wurden 


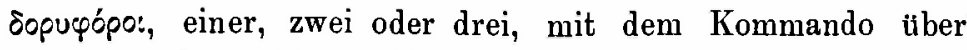
kleinere oder größere Abteilungen von u $\pi \alpha \sigma \pi \tau \sigma \tau \alpha \ell$ betraut (BP II, 19 p. 234, 15; BV I, 16 p. 380, 1; I, 19 p. 389, 24; I, 23 p. 401,20 ; BG I, 27 p. 126,3 ; p. 127,5 ; II, 2 p. 150 , 3 ; II, 7 p. 176,15 u. a. m.). Mitunter wurden sie auch an die Spitze von Reichstruppen gestellt (BP I, 9 p. 45, 12 ; BV II, 19 p. 494,1$)$ oder kaiserlichen Offizieren beigegeben (BP II, 20 p. 243,11 ; BG II, 4 p. 160,5 ; III, 11 p. 320,14 ; III, 15 p. 337,16 ; III, 28 p. 395,15 ; III, 30 p. 402, 2) und übten vielleicht eine gewisse Kontrolle über die letzteren aus. Je größBer das Gefolge eines Generals war, desto selbständiger und glänzender war seine Stellung. Narses stützte sich bei seiner Opposition gegen Belisar nicht zum wenigsten auf sein Gefolge (BG II, 18 p. 218,6), und daß Belisar mit seinen 7000 Reitern dem Kaiser unbequem war, liegt auf der Hand. Solche Position zu erreichen war das Motiv für die Generale, die erheblichen Opfer für das Gefolge zu bringen. Allerdings verstanden sie es, sich durch Bedrückung der Einwohner (BG III, 1 p. 283, 13; IV, 16 p. 541, 2), worin namentlich Bessas Außserordentliches leistete (BG III, 17 p. 347, 13; 19 p. 356, 16 ; 20 p. 360,7 ; IV, 13 p. 525, 11), schadlos zu halten. Belisar machte eine Ausnahme, hielt streng darauf, daß die Einwohner geschont wurden (BV I, 16 p. 378, 14; 17 p. 382, 10; BG I, 10 p. 54,6 ; II, 8 p. 181,6 ; III, 1 p. 281,15 ), und war von der größten Freigebigkeit gegen seine Soldaten. Verwundeten spendete er Geld; Tapferkeit belohnte er durch Schenkung von Armbändern und Halsketten, und verlorene Pferde und Waffen, die Eigentum der Leute waren, ersetzte er (BG III, 1 p. 281, 10; vgl. BG I, 28 p. 131, 14).

Das Gefolge war eine Haustruppe des Gefolgsherrn, es gehörte zu seiner oixía. Die Belisars wird wiederholt erwähnt

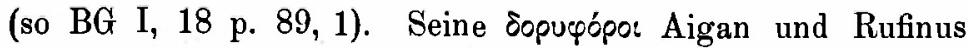

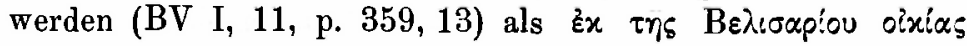

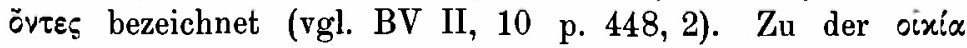
gehörte aber auch eine zahlreiche Dienerschaft, die als $0 \pi \alpha \delta 0^{i}{ }^{3}$ ) bezeichnet wird und einen eigenen Vorsteher hatte. Unter

3) Daß auch die sopụópor gelegentlich ò $\pi \alpha 8$ of genannt werden, ist oben S. 116 bemerkt. 
Belisar erwähnt Procop (BG III, 36 p. 434, 16) einen solchen

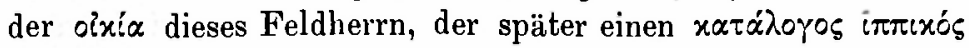
kommandiert. Unter Narses nennt Agathias (I, 19 p. 55, 17)

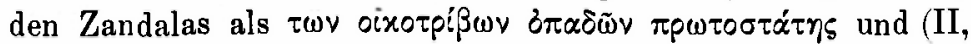

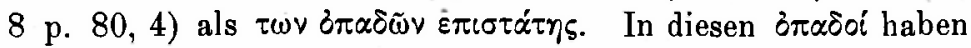
wir die höhere Dienerschaft zu erkennen, die niedere wird

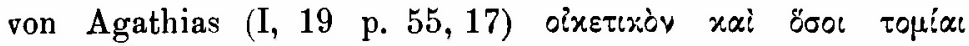

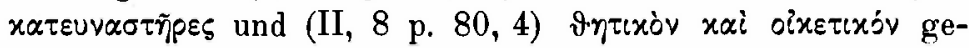
nannt. Aus letzterer Stelle erhellt auch, dafis diese Leute, soweit sie kriegstüchtig waren, am Kampfe teilnahmen. Dies war ursprünglich germanische Sitte (Mommsen Hermes 24 p. 244 A. 1). Daßs der General auch für die Unterhaltung der Dienerschaft die Kosten zu tragen hatte, zeigt Agath. IV, 22 p. 252, 14), wo jener oben S. 117 erwähnte Geschäftsmann

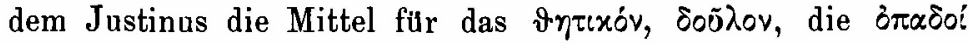

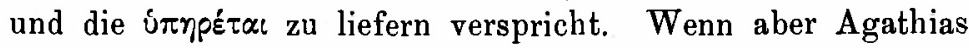
(I, 19 p. 55, 12) zu dem Gefolge des Narses auch $\tau \eta_{5}$ ápy. $\gamma_{5}$

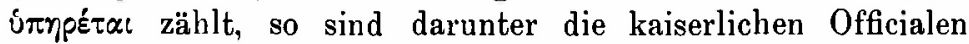
seines Bureaus zu verstehen, die natürlich vom Kaiser besoldet wurden. Der hienach schon ziemlich starke Trof des Heeres wurde dadurch noch vergröfert, daf manche Soldaten männliche und weibliche Sklaven hatten (BG I, 25 p. 119,9) und zahlreiche Lasttiere erforderlich waren, um diese Dienerschaft sowie die Waffen und Kleider der Mannschaften zu befördern (BG III, 11 p. 323, 8).

\section{Die Flotte.}

Von der Kriegsflotte ist nur einige Male die Rede. Offenbar war das Interesse der Regierung für dieselbe nicht groß. Als Kaiser Leo im J. 468100000 Mann gegen Geiserich schickte, wurden die Schiffe aus dem ganzen Orient zusammengebracht, und das verursachte enorme Kosten, die gänzlich verloren waren, da die Flotte durch Brander der Vandalen vernichtet wurde (BV I, 6 p. 335, 14 und p. 339). Belisar führte neben 500 Transportschiffen, die mit 20000 ägyptischen, ionischen und cilicischen Seeleuten bemannt waren, und von denen die größten 50000 Scheffel (= 2125 Tonnen), die kleinsten 3000 Scheffel (=127 Tonnen) fafiten, 92 Schlacht- 
schiffe nach Afrika. Diese, Dromonen genannten, Fahrzeuge waren Schnellsegler mit nur einer Ruderreihe und einem Verdeck zum Schutze der Ruderer. Wenn Procop meldet, diese 92 Schiffe hätten eine Besatzung von 2000 Mann, zugleich Seesoldaten und Ruderer, gehabt, so ist das wohl ein Irrtum, da auf jedes Schiff nur 21 oder 22 Mann gekommen wären (BV I, 11 p. 360) ${ }^{4}$ ). Die Dromonen werden kaiserliche Schiffe gewesen sein, Privatschiffe dagegen die Transportfahrzeuge. Leider erfahren wir nicht, ob das Schiff, mit dem Belisar später von Sicilien nach Karthago fuhr, und auf dem er 100 sopu-

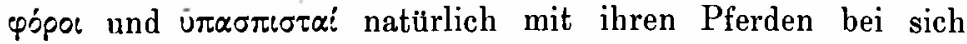
hatte, ein Dromon oder ein Transportschiff war (BV II, 15 p. 476, 11). Von Einzelheiten dürften folgende interessiereu. Als die Flotte von Byzanz nach Afrika abgehen sollte, legte das Admiralschiff vor dem kaiserlichen Palaste an, der Bischof Epiphauius sprach ein Gebet und ließ einen eben getauften Soldaten einsteigen (BV I, 12 p. 362, 20). Um die Flotte bei Tag und Nacht zusammenzuhalten, ließ3 Belisar auf dem Admiralschiffe und zwei anderen Schiffen, auf denen seine Dienerschaft fuhr, die Segel von der oberen Spitze bis zum dritten Teile rot anstreichen und am Hinterteile Laternen aufstellen (BV I, 13 p. 366, 12). Die Fahrt von Byzanz nach Afrika dauerte drei Monate (BV I, 15 p. 377, 11). Als Belisar im J. $535 \mathrm{zum}$ Gothenkriege nach Sicilien gesegelt war, legte er im Hafen von Palermo an. Den Angriff auf die Stadt eröffnete er mit einem eigentümlichen und erfolgreichen Verfahren. Da die Masten seiner Schiffe böher waren als die Stadtmauern, ließ er seine Bogenschützen in die Boote steigen und diese bis zur Höhe der Masten aufwinden (BG I, 5 p. 28, 7). Ein ähnliches Verfahren, das die Römer gegen die Perser bei der Belagerung der Stadt Phasis anwandten, erwähnt Agathias (III, 21 p. 185, 20).

Von einer Seeschlacht bei Ancona mit den Gothen berichtet Procop (BG IV, 23 p. 577). Hier haben die Gothen 47 Kriegsschiffe, die Römer anfangs 48, später 60 . Jene ver-

4) Nach Constant. Porphyr. De caerem. II, 45 p. 670, 3 Ed. Bonn. soll ein Dromon als Besatzung 70 Seesoldaten und 230 Seeleute haben die im Notfalle auch als Soldaten verwandt werden können. 
stehen nicht zu manövrieren und unterliegen kläglich; nur 12 ihrer Schiffe entkommen. Aehnlich erging es nach Agathias (V, 21 f. p. 325; 327) den Hunnen, die zum Angriff auf die Chersones aus zusammengebundenem Rohr eine elende Flotte gebaut hatten.

Von Flußsschifffahrt ist nur einmal die Rede. Eine römische Flotte kommt im Gothenkriege nach Ostia. Es kommt darauf an, die Lebensmittel nach Rom zu schaffen. Man ladet sie auf Boote, die man mit Brettern vor den feindlichen Geschossen schützt (BG II, 7 p. 172 f.).

\section{Varia.}

\section{A. Die Schutzwaffen.}

1. Helme werden natürlich oft erwähnt; es fehlt aber gänzlich an Anhaltspunkten, die Form zu bestimmen.

2. Panzer. Wenn Agathias (II, 8 p. 80, 11) sagt, das erste Glied der römischen Infanterie $\left(\pi \rho \omega \tau \circ \sigma \tau \alpha^{\prime} \tau \alpha\right)$ habe $\vartheta \dot{v} \rho \alpha \alpha_{5}$ $\pi c \delta$ jpess getragen, so kann damit nur die lorica hamata (Kettenpanzer) gemeint sein. Auf einen Plattenpanzer aus Metall lassen vielleicht einige Stellen schließen. BP I, 18 p. 94, 9 wird erzählt, die persischen Pfeile sprängen, ohne Schaden zu tun, von den Brustharnischen der Römer ab; allerdings schiebt Procop die Schuld dafür auf die schlechten Bogen und den Mangel an Kraft bei den Persern. BP II, 25 p. 266, 21 befinden sich die römischen Kavalleristen auf eiliger Flucht vor den Persern und werfen den Panzer weg, gewiß um nach Beseitigung der schweren Last schneller vorwärts zu kommen.

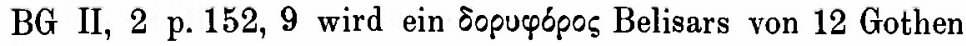
umzingelt, die mit Lanzen auf ihn einstoßen; aber ohne Erfolg, da der Panzer Stand hält. Agathias spricht IV, 22

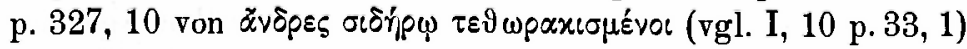

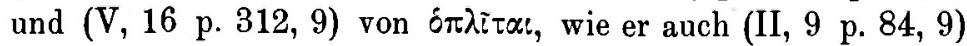

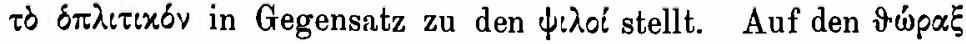

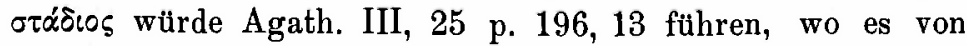

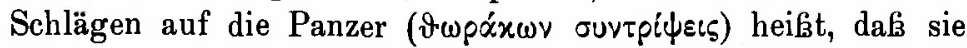
großen Lürm machten.

3. Schilde. Von den mit Schilden üblichen Operationen ist mehrfach die Rede, so von Deckung gegen Kavallerie- 
augriffe (BG IV, 29 p. 612, 11; Agath. II, 8 p. 80, 12), von Schutz einzelner Personen (BG IV, 11 p. 554,6 f.), von Deckung gegen von oben kommende Pfeile (Agath. V, 1

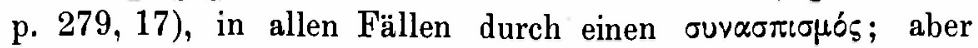
über die Gestalt der Schilde sowie über das Material, aus dem sie hergestellt waren, finden wir nichts. Keinenfalls kann aus der Notiz, daß3 aufschlagende Pfeile großen Lärm verursacht haben (BG IV, 11 p. 514, 8; 29 p. 612, 16) auf Holz oder Metall geschlossen werden. Eher liefe sich daraus, dak ein Offizier stürzt und dabei sein Schild zerbricht (Agath. IV, 18 p. 245,4 ), auf Holz schließen. Die Reiter haben $\pi \dot{\lambda} \lambda \tau$ : (Ag. II, 8 p. 80,$2 ; \mathrm{V}, 19$ p. 319,16$)$.

4. Beinschienen haben die Bogenschützen (BP I, 1 p. 12, 9), darum fällt es Agathias (II, 5 p. 74, 1) auf, daß die Franken keine Beinschienen kennen. Näheres ist nicht $\mathrm{zu}$ ermitteln.

5. Kleidung. BV II, 23 p. 511, 20 ist die Rede von einer Kriegslist, durch die allerlei Leute als Soldaten verkleidet werden und zu diesem Ende die $\{\mu \alpha \dot{\alpha} \tau \mid \alpha$ erhalten, $\alpha$ 'P $\omega \mu \alpha i \omega \nu$

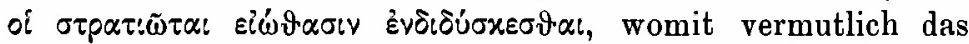
Sagum gemeint ist. Ebenfalls dieses oder die Paenula ist zu

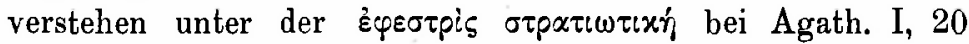

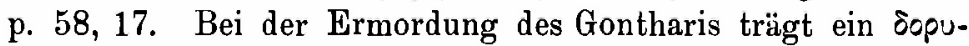
ழópos des Artabanes das nackte Schwert unter der Achsel, verborgen durch die $\varepsilon \pi \omega \mu i^{\prime}$ (BV II, 28 p. 530, 15). Hieraus ist zu entnehmen, daß unter è $\pi \omega \mu$ ís nicht jener kurze Mantel zu verstehen ist, der in klassischer Zeit mit diesem Worte bezeichnet wird, sondern ein längerer.

\section{B. Angriffswaffen.}

Lanze und Dolch, Schwert und Wurfspie $B$ sind durchaus ublich. Das Pilum war nicht mehr in Gebrauch, sonst würde Agathias (II, 5 p. 74, 11 f.) den bei den Franken üblichen, mit jenem fast identischen, Angon nicht so genau beschrieben haben.

Die BG I, 19 p. 140, 10 erwähnten $\dot{\alpha} x o^{\prime} \tau \tau \alpha^{\prime} \mathrm{J} \sigma \alpha \cup \rho: x \dot{\alpha}$ müssen kurze Lanzen gewesen sein. Ein Isaurier hat dort in jeder Hand eine derselben, wirft sie jedoch nicht ab, sondern 


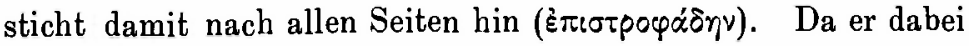
den Schutz des Schildes entbehrt, wird er vielfach verwundet. Uebrigens nennt Agathias (III, 20 p. 185, 1) die Isaurier

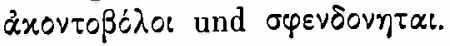

Den größten Wert legte man in Justinians Zeit auf den Gebrauch des Bog e n s. Die Schlacht begann mit dem Bogenkampfe, und die Pfeile flogen so dicht, daß sie die Luft verdunkelten (BP I, 14 p. 71, 10; Agath. III, 25 p. 195, 1). Waren die Pfeile verschossen, so folgte der Nahkampf (BP I, 14 p. 71,3 und 16). Manche Leute, die jenen Fernkampf nicht hochschätzten, nannten zum Spott die Soldaten rołóra: (BP I, 1 p. 11,9). Man hatte Bogenschützen zu Fuf, deren einmal (BG IV, 32 p. 621, 22) 8000 erwähnt werden, und zu

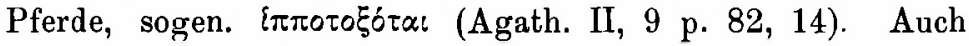
die höheren Offiziere verstanden das Bogenschießen. Ein Dux erschof den Stotzas (BV II, 24 p. 514, 11), und Belisar tötete mit Meisterschüssen zwei vornehme Gothen (BG I, $22 \mathrm{p}$. 105, 15). Ausführlich spricht Procop (BP I, 1 p. 12, 8) über die $i \pi \pi \circ \tau \circ \xi$ ó $\alpha$. Sie tragen Panzer und Beinschienen bis zum Knie; an ihrer rechten Seite hängen die Pfeile, an der linken das Schwert; bei einigen hängt am Pferde auch eine Lanze; auf den Schultern liegt ein kleiner, kragenartiger Schild ohne Handhabe, der den Nacken und zum Teil das Gesicht schützt. Sie reiten vortrefflich und verstehen es, bei schnellster Gangart des Pferdes nach vorwärts und rückwärts zu schießen. Dadurch, daß sie die Bogensehne bis nach dem rechten Ohre zuräckziehen, geben sie dem Pfeile die rechte Kraft, die den von den körperlich schwächeren Persern abgegebenen Schüssen

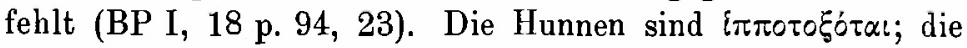
Bogenschützen der Gothen sind Infanteristen, ihre Reiter kämpfen nur mit Lanze und Schwert (BG I, 27 p. 129, 9). Doch ist wiederholt von besonders kräftigen Schüssen einzelner

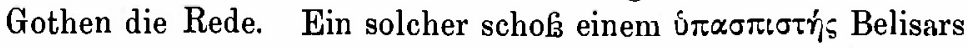
zwischen Nase und Auge einen Pfeil in den Kopf, der bis zum Nacken durchdrang (BG II, 2 p. 151, 15); ein anderer schoß auf einen römischen $\tau \alpha \xi$ : $\alpha$ p 05 und durchbohrte den Schild und den Metallpanzer (Agath. I, 9 p. 32, 18). Im Gegensatze zu den Gothen waren die Vandalen schlecht im Fuf- und Fern- 
kampfe mit Wurfspiefi und Bogen (BV I, 8 p. 348, 22), sie waren Reiter und bevorzugten Lanze und Schwert. Gelimer befahl einst, nur mit dem Schwert zu kämpfen (BV II, 3 p. $421,8)$. Interessant ist was von einer Pfeilwunde erzählt wird,

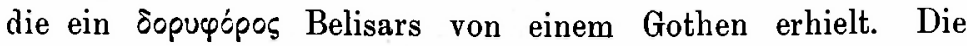
Pfeilspitze drang zwischen Auge und Nase ein, während der schlecht befestigte Schaft auf die Erde fiel. Die im Kopfe befindliche Spitze behinderte den Mann in keiner Weise; nach 5 Jahren kam sie allmählich heraus, und der Verwundete hatte die Hoffnung, mit der Zeit ganz von ihr befreit zu werden (BG II, 5 p. 167, 3).

\section{Feldzeichen.}

Der Feldherr hatte ein besonderes Feldzeichen. BV II, 2 p. 415, 20 wird dasjenige Belisars $\beta \alpha^{\prime} v \delta o v^{5}$ ) genannt. BV II, 10

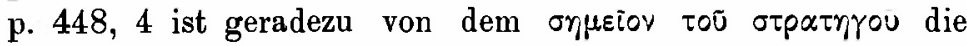
Rede. Dieses in der Schlacht zu tragen ist eine Ehre für den mutigsten Mann, hier den Thraker Rufinus, der zum Gefolge Belisars gehörte (BV II, 11 p. 359, 14). Er wird als $\beta \alpha y-$

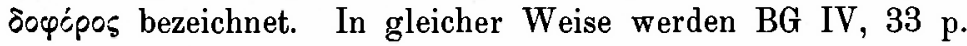
633, 6 die $\sigma \eta \mu \varepsilon i \alpha$ des Narses und Johannes erwähnt. BG II 17 p. 216, 4 erkennen die Gothen an den Feldzeichen, daß Belisar persönlich anwesend ist.

Je energischer die Truppen ihre Fahnen zu verteidigen pflegen, desto betrübender war der Verlust derselben, von dem einige Male berichtet wird. BV II, 17 p. 488, 10 nehmen die Aufrührer unter Stotzas der Abteilung des Johannes alle Feldzeichen ab. BV II, 28 p. 533, 16 waren in einem Kampfe mit einem maurischen Stamme die Feldzeichen verloren gegangen, wurden aber später wiedergewonnen und nach Byzanz geschickt. BG III, 4 p. 298, 8 erobern die Gothen sämtliche Feldzeichen. BG III, 40 p. 456, 13 und 23 gehen im Kampfe mit den Sclaveni die Feldzeichen verloren, werden aber später wieder gefunden.

Bekanntlich genossen in älterer Zeit die Feldzeichen göttliche Verehrung und standen in einer aedicula. Das hörte natürlich in christlicher Zeit auf, indessen wurden doch noch

5) Lat. bandum. Du Cange Gloss. m. e. i. Latin. s. v. "bandum vexillum; signum militare". 
besondere Lokale für dieselben gebaut. Justinian ließ ein solches zu Zenobia am Euphrat errichten (De aed. II, 8 p. 234,10 ).

Auf dem Marsche wurden die Feldzeichen, wie für frühere Zeit aus Cichorius, Trajanssäule Taf. 4 und $5 \mathrm{zu}$ ersehen ist, auch jetzt noch hinter dem Feldherrn zusammen den Truppen vorangetragen. Wenigstens erzählt Agathias (III, 24 p. 194, 12), daks bei einem feierlichen Kirchgange von 5000 Mann die Feldzeichen unmittelbar hinter dem Kommandanten ihren Platz hatten.

Ein eigentümliches Verfahren wird von Belisar berichtet (BP I, 14 p. 67, 16). Er ließs vor der Schlacht die mit den Persern erfolglos über den Frieden gewechselten Briefe an ein Feldzeichen heften, um ihnen ihre Treulosigkeit vorzuhalten. In ähnlicher Weise hatten die Ephthaliten (Hunnen) das Salz an ihre Fahne geheftet, das der König Perozes beim Ableisten des von ihm später gebrochenen Friedenseides in der Hand gehabt hatte (BP I, 4 p. 20,13). Es scheint sich um eine persische Sitte $\mathrm{zu}$ handeln.

Ins Gebiet der Fabel gehört folgende` Erzählung. Die Feldzeichen der Besatzung von Antiochia standen mit der Front nach Westen gerichtet, drehten sich dann nach Osten und endlich wieder nach Westen. Man sah darin ein Vorzeichen davon, daß die Herrschaft über die Stadt auf die Perser übergehen würde (BP II, 10 p. 195, 1).

Ueber die Gestalt der signa erfahren wir nichts. Es ist jedoch nach dem, was ich Phil. LXIV p. 609 ff. ausgeführt habe, wahrscheinlich, daß nur vexilla und dracones üblich waren. Vom Adler ist nirgends die Rede, die Feldherrnfahne war vermutlich ein vexillum wie das labarum Constantins (s. Baumeister, Denkmäler Fig. 439 b).

\section{Signalinstrumente.}

Signale mit der $\sigma \alpha^{\prime} \lambda \pi \iota \gamma \xi$ (= tuba) werden mehrfach erwähnt. So BV I, 14 p. 372,12 ; II, 20 p. 499, 14; BG I, 10 p. 50,8 und p. 52, 15; Agath. III, 27 p. 201, 9; IV, 17 p. 244, 9; IV, 19 p. 246, 14. Bei der Bestürmung der Stadt Phasis

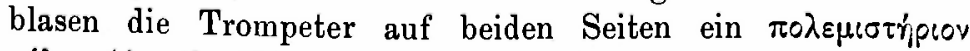

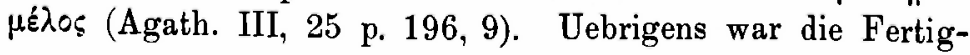


keit der Trompeter in Behandlung ihres Instrumentes nur noch gering. Nach Vegetius II, 22 wurde das Signal sowohl zum Angriff als zum Rückzuge mit der tuba gegeben. Zu Belisars Zeit verstanden das die Bläser nicht mehr, und man scheint sich für den Rückzug auf Rufen beschränkt zu haben (BG II, 23 p. 241,20). Als sich dieses Mittel einst nicht bewährt hatte, riet Procop, das Angriffssignal durch die $\sigma \alpha \dot{\lambda} \pi i \gamma \gamma \varepsilon \varsigma$ $\{\pi \pi i x \alpha i$, das Rückzugssignal dagegen durch die $\sigma \dot{\lambda} \lambda \pi \imath \gamma \gamma \varepsilon \varsigma \pi \epsilon \zeta \imath x \alpha i$ geben zu lassen. Die ersteren waren Instrumente aus sehr dünnem Holze mit einem Lederüberzuge (vgl. Baumeister, Denkmäler p. 1658), die letzteren aus starkem Metall (BG II, 23 p. 241, 24). Ueber die Gestalt des Kavallerieinstrumentes erfahren wir nichts, das der Infanterie war die tuba.

\section{E. Exerzieren.}

Im Frühling 553 ließ̧ Narses die Truppen, welche in den Winterquartieren nicht geübt hatten, fleißig exerzieren. Es werden drei Uebungen genannt: $\tau \rho \circ \chi \alpha \dot{\alpha} \zeta e c \nu, d$. h. größere Eilmärsche in Waffenrüstung machen (vgl. Polyb. 10, 20; Suid. s. v.), aufs Pferd springen, worauf um so größeres Gewicht gelegt wurde, als der Gebrauch des Steigbügels unbekannt war,

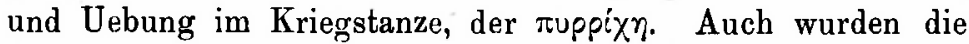
Leute daran gewöhnt, genau auf die Signale zu hören (Agath. II, 1 p. 64,2 ).

\section{F. Eintritt in das Heer.}

Der Eintritt in das Reichsheer geschah meist freiwillig. Germanus (BV II, 16 p. 484, 15) hält seinen Leuten vor, sie seien vom Lande mit dem Ranzen und einer einzigen Tunika nach Byzanz gekommen und vom Kaiser in ihre jetzige Stellung gebracht. Schon oben S. 108 ist erwähnt, daß̧ der nachmalige Kaiser Justinus mit zwei Kameraden, um der drückenden Armut zu entgehen, mit etwas Zwieback und einer Decke zu Fußs nach Byzanz kam. Dort wurde er in einen $x \alpha \tau \alpha \dot{\lambda}$ oyos eingestellt, bald aber seiner Schönheit wegen in die Scholae aufgenommen, deren Kommandeur er später wurde, - ein Beweis, daf dem Avancement des gemeinen Mannes nichts im Wege stand (Hist. arc. 6 p. 43,2). Von einer Einstellung von Mannschaften als Steuerleistung ist bei Procop und Agathias 
nirgends die Rede, wohl aber von Werbungen. Solche des Germanus in Thrakien und Illyrien sind schon oben S. 108 erwähnt. Hier fügen wir hinzu, daß dieser, der nach BG III, 39 p. 447, 7 für sein eigenes Geld eine Menge Truppen an sich zog, außer für das Reichsheer auch für sein Gefolge gesorgt haben wird. Ob Belisar (BG III, 10 p. 315, 24) in Thrakien für den Kaiser oder für sich warb, wird nicht angegeben.

\section{G. Verpflegung.}

In höchster Instanz wurde das Verpflegungswesen und die Soldzahlung durch Kriegskommissare geleitet, vornehme Zivilisten, die dem militärischen Kommandeur beigegeben wurden. So ernannte Anastasius den Aegypter Appion zum xopnrós im Perserkriege und verlieh ihm zu besserer Lösung seiner Aufgabe kaiserliche Gewalt (BP I, 8 p. 40,8). Im Perserkriege

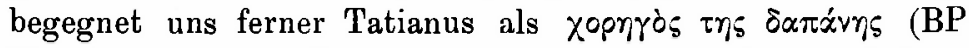
II, 10 p. 195,9). In Afrika bekleidete dieses Amt Archelaus,

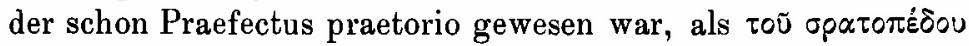

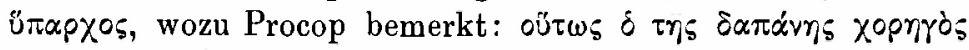

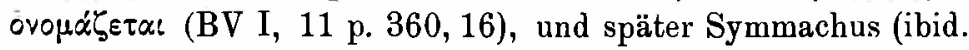
II, 16 p. 48:, 20). Eine andere Bewandtnis hatte es mit der Stellung des Rusticus (Agath. III, 2 p. 140, 2). Dieser war Vertreter des Comes sacrarum largitionum im Colcherlande. Seine Aufgabe war genaue Berichterstattung über alle Vorkommnisse an den Kaiser und Verteilung von Belohnungen an die Truppen. Als sein Nachfolger wird Johannes Dacnas genannt (Agath. IV, 17 p. 242, 11). Mit der Verpflegung der für den Vandalenkrieg bestimmten Truppen war der Praefectus praetorio Orientis Johannes aus Cappadocien (BP I, 24 p. 121, 15), ein ungebildeter und schlechter Mensch, beauftragt. Des Geldgewinns wegen gab er den Leuten schlecht gebackenes Brot, dessen Genuß Krankheiten und viele Todesfälle verursachte. Als die Flotte bei Methone anlegte, befahl Belisar, dak das Brot aus dem Lande bezogen werden sollte (BV I, 13 p. 368). Hier noch einige Einzelheiten. Auf der Chersones, nicht weit von Gallipoli lieł Justinian Weinkeller und Kornmagazine für die Soldaten erbauen (De aed. IV, 11 p. 302, 12). - Vierhundert Römer, die im Grabmal Hadrians einge- 
schlossen waren, hatten einen Tag und eine Nacht keine Lebensmittel gehabt, da machten sie sich mit dem Gedanken vertraut, Pferdefleisch zu essen, konnten sich aber, selbst nachdem sie noch einen Tag gehungert hatten, nicht dazu überwinden, sondern machten sich zum Todeskampfe bereit (BG III, 36 p. 435, 8). - Während die Perser abends zu essen pflegten, nahmen die Römer im Felde ihre Mahlzeit, das äpiotov (BG II, 2 p. 150, 12), mittags ein (BP I, 14 p. 71, 7; II, 18 p. 231, 8). Eine römische Abteilung hat 800 Perser vernichtet und bereitet nun sorglos das äpırov, wobei es Fleisch gibt, wird aber von dem Hauptheere der Perser iuberfallen und flieht, ohne gegessen zu haben (BP I, 8 p. 41, 15). Vor der Schlacht bei Taginae fürchtet Narses einen Ueberfall der

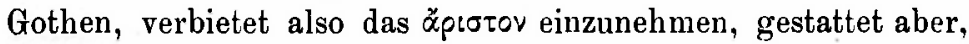
in Reih und Glied und in voller Rüstung etwas Wein und

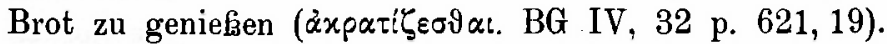

\section{H. Wertschätzung der Truppenteile.}

Aus der oben gegebenen Uebersicht geht hervor, welch große Bedeutung das barbarische Element im römischen Heere hatte. Procop (BG I, 1 p. 6, 11) beklagt das bitter. Je kräftiger, meint er, sich die Barbaren zeigten, desto mehr verschwände das Ansehen der römischen Truppen. Letztere wurden vielfach Гpasxoí genannt. Einfach und ohne Tadel lesen wir diese Bezeichnung BV II, 27 p. 527, 8. Offenbare Verachtungr liegt aber in derselben, wenn die Gothen sie gebrauchen, wie BG I, 18 p. 93,3 ; I, 29 p. 136, 4; III, 21 p. 366, 10; IV, 29 p. 581, 13, wo auch der Zusatz àvavঠิpot gemacht wird. Daf sogar kaiserliche Beamte keinen Anstand nahmen, die Reichstruppen so $\mathrm{zu}$ benennen, werden wir unten sehen. Ist

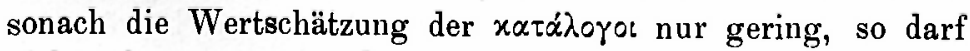
nicht übergangen werden, dał zweimal die Tapferkeit derartiger Abteilungen besonders gerühmt wird. BG IV, 29, p. 611,22 f.; Agath. II, 10 p. 85, 4.

In ähnlicher Weise verachteten innerhalb des Reichsheeres die Kavalleristen ihre Kameraden von der Infanterie. Auf die Reiterei wurde in Justinians Zeit das größte Gewicht gelegt. Schon Vegetius $(I, 20)$ sagt, die Römer hätten nach 
dem Vorbilde der Gothen, Alanen und Hunnen ihre Reiterei sehr vervollkommnet. Je mehr nun die Zahl der barbarischen Heeresabteilungen zunahm, desto höher stieg die Wertschätzung dieser Waffe. Die oben genannten barbarischen Hilfsvölker waren Reiter; beritten war auch der größte Teil der Foederati sowie das Gefolge der Generale; dazu kamen noch die Reiter Ė $x \alpha \tau \alpha \lambda$ óyou. Dem entspricht der starke Prozentsatz der Kavallerie in dem gegen die Vandalen geschickten Heere, wo auf 10000 Mann Fufrolk 5000 Reiter kommen (BV I, 11 p. 358, 18), und daß im Vandalenkriege eine Schlacht nur von Reitern ausgefochten wurde (BV II, 1 p. 415, 1). Auch im Gothenkriege wollte Belisar einst nur mit Kavallerie kämpfen, zumal sich viele Infanteristen mit erbeuteten Pferden beritten gemacht hatten - was auch BG III, 18 p. 352, 6 vorkommt - sah aber auf die Vorstellungen verschiedener Offiziere davon ab (BG I, 28 p. 132, 19).

Das Urteil ïber die Infanterie, welche fast ausschließlich

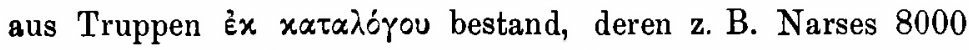
Mann hatte (BG IV, 31 p. 618, 11), war sehr ungünstig. Man meinte, schon beim ersten Zusammenstok mit dem Feinde pflege sie zu fliehen (BG I, 28 p. 133, 2), und ihre Kommandeure freuten sich ihres Berittenseins besonders deshalb, weil sie dadurch auf der Flucht besser führen (ibid. v. 17). So erklärt es sich leicht, daß3 400 römische Reiter, denen Totilas zur Wahl verstellte, ob sie nach Ablieferung ihrer Waffen und Pferde nach Byzanz gehen, oder lieber zu den Gothen ubertreten wollten, die letztere Alternative vorzogen, weil sie sich schämten, den Weg zu Fuß und ohne Waffen zu machen (BG III, 36 p. 436, 5). Indessen mußsten in den Wechselfällen des Krieges mitunter Reiter absitzen. So BP I, 18 p. 96, 13, wo die durch die persische Reiterei arg bedrängten Leute sich durch festen Anschluf der Schilde mit Erfolg verteidigten, und $\mathrm{BG} I V, 31$ p. 618,15 , wo die nicht recht zuverlässigen Langobarden und Heruler zu Fuß mit der römischen Infanterie aufgestellt wurden.

\section{Mißbräuche im Heerwesen.}

Ueber arge Mikstände in der Verwaltung des Heeres be- 
lehrt uns Procop in der Historia arcana 24 p. $132 \mathrm{ff}$. Sie hatten ihren Grund darin, daf Justinian für seine zahlreichen Bauten sowie für die an die Barbaren zu leistenden bedeutenden Zahlungen auferordentlich viel Geld nötig hatte. Es wurde daher am Militärwesen gespart und der Soldat in seinen Bezügen verkürzt. Die zur Besorgung der Finanzangelegenheiten bestimmten Beamten, die Logotheten, verstanden es meisterhaft, die Soldaten zu schädigen, zumal sie von allen ersparten Geldern den zwölften Teil erhielten. Nun wurden die Truppen nach dem Dienstalter besoldet und rückten je nach Stellenerledigung in bessere Bezüge vor. Die Logotheten tilgten jedoch in den Stammrollen ${ }^{6}$ ) die Namen der Ausgeschiedenen nicht, so daf die jungen Soldaten höhere Löhnung nicht bekommen konnten. Neue Verzeichnisse legten sie nie an. Infolge dessen waren die Truppenkörper niemals vollzählig, aber der Kaiser sparte viel Geld. Ferner warfen die Logotheten manchen Soldaten vor, sie seien $\Gamma_{p}$ alxoi, als ob aus dem griechischen Osten kein anständiger Soldat kommen könnte. Auch beschuldigten sie mitunter Leute, ihre Papiere seien gefälscht, und sie seien eigentlich gar nicht berechtigt im Heere zu dienen. Andere wiesen sie zurück, weil sie sich mehrere Tage vom Lager fern gehalten hätten.

$\mathrm{Zu}$ den Logotheten gesellten sich ebenfalls als Quäler und Bedrücker Scholarii, welche in allen Teilen des Reiches die Soldaten auf ihre Diensttüchtigkeit prüften. Die Untauglichen wurden ohne Pension entlassen und mujiten betteln gehen. Wer dazu im Stande war, kaufte dieses Schicksal mit vielem Gelde $a b$, das den Beamten zufloß. Dasselbe Verfahren wurde gegen die Foederati eingeschlagen. Nicht besser ging es den Limitanei, den Grenzern. Diese bekamen zunächst vier oder fünf Jahre hindurch ihre Kompetenzen nicht und wurden schließlich ganz aus der Armee gestrichen. Auch sie sahen sich auf die Güte Mildtätiger angewiesen.

Ebenso schändlich war das Verhalten gegen die Scholarii. Diese waren früher eine angesehene, zum Dienste im

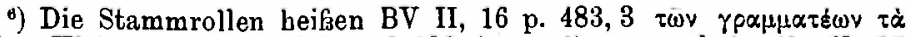

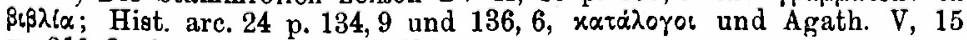
p. $310,2 \tau \dot{\alpha} \tau \omega v x \alpha \tau \alpha \lambda \delta \gamma \omega \vee \beta \varepsilon \beta \lambda l \alpha$. 
Palast des Kaisers bestimmte Truppe, die auch besser bezahlt wurde, als die andern Soldaten. Bis auf Zeno nahm man dazu besonders tüchtige Leute aus Armenien. Dieser Kaiser stellte jedoch allerhand Leute, auch kriegsuntüchtige, ein, wofür er sich gehörig bezahlen ließ3. Und dabei blieb es. Schließslich konnten sich auch Sklaven auf diese Weise eine Rente kaufen. Unter Justinus' Regierung fand es Justinian angemessen, den 3500 Scholarii noch 2000 Ueberzählige hinzuzusetzen, wodurch viel Geld einkam. Als er aber selbst zur Regierung gelangt war, beseitigte er diese Supernumerarii, gab aber die gezahlten Beträge nicht zurück. Gegen die Scholarii älterer Ordnung verfuhr er anders. Er drohte diesen ganz unkriegerischen Leuten, er wolle sie in den Krieg schicken; um das zu vermeiden, verzichteten sie zu Gunsten des Kaisers auf ihren Sold. Ebenso erging es den Domestici und Protectores. Ursprünglich waren diese ein Offizierkorps, dessen Mitglieder in besonderen Aufträgen verwandt wurden, aber niemals einen Truppenkörper bildeten. Sie standen höher als die Scholarii und bezogen höhere Kompetenzen, mußiten zu Justinians Zeit allerdings ihre Stellen auch höher bezahlen. Sie waren damals völlig unkriegerisch und wurden lediglich nach ihrer sozialen Stellung zugelassen. Auch diesen drohte Justinian Sendung in den Krieg an, worauf sie auf ihre Besoldung verzichteten. Der gemeine Soldat wurde auch dadurch geschädigt, daß die alte Sitte, nach der jeder Mann alle fünf Jahre ein Geschenk von 5 Goldstücken erhielt, unter Justinian zunächst nicht geübt wurde und während seiner langen Regierung zuletzt völlig in Vergessenheit geriet.

Schädliche Maßregeln waren noch die Aufhebung der Exploratores, Spione, welche Nachrichten über die Pläne und Operationen der Feinde gebracht hatten (BP I, 21 p. 108, 15; Hist. arc. 30 p. 163, 3), sowie die Abschaffung der vom Staate erhaltenen lasttragenden Kamele, welche die Lebensmittel getragen hatten (Hist. arc. 30 p. 163, 15).

Von diesen Miß̧bräuchen redet auch Agathias (V, 13 p. 305 f.), namentlich hebt er hervor, wie sehr der Bestand des Heeres unter Justinian verringert worden sei. Die alten Kaiser hätten 645000 Mann unter den Fahnen gehabt (jedenfalls 
denkt der Schriftsteller hier an das fruhere Doppelreich). Diese Zahl sei aber auf 150000 reduziert. Justinian habe in seinem Alter keine Freude mehr am Kriegführen gehabt und es vorgezogen, die Reichsfeinde unter sich zu verhetzen, und daher habe er nicht mehr so viele Truppen nötig gehabt. Von den Betrügereien der Beamten, Unterschlagung und zu später Auszahlung der Gelder und Auslieferung der Lebensmittel, schließlich auch von aus nichtigen Gründen geforderter Rückzahlung erhaltener Gelder erzählt Agathias dasselbe wie Procop.

\section{Zustände im Heere.}

Daß̧ sich unter diesen Umständen in der Armee sehr unerquickliche Zustände herausbildeten, versteht sich von selbst. Besonders verursachte das Ausbleiben des Soldes, den Justinian, ohne sich zu schämen und ohne die Folgen zu fürchten, schuldig blieb (Hist. arc. 22 p. 124, 6), und der auch noch zu Narses' Zeit, obwohl sich dieser bei Uebernahme des Kommandos Tilgung der Rückstände und gewiß auch pünktliche Zahlung ausbedungen hatte (BG IV, 26 p. 597, 18), unregelmäß̧ig ausgezahlt wurde (Agath. I, 18 p. 52, 10), mancherlei Unordnungen. So ist wiederholt die Rede davon, daß die Truppen nicht kämpfen wollen (BG III, 1 p. 284, 4 ; III, 6 p. 302, 2 und 6; III, 12 p. 324, 20). In Byzanz kam es zu Straßenunfug der Soldaten (Hist. arc. 22 p. 126, 10). Die Illyrier zogen einst eigenmächtig ab (BG III, 11 p. 320, 19). Nicht selten waren Desertionen (Agath. V, 14 p. 307, 15). Durch den Hinweis auf nicht gezahlten Sold wurden in Afrika viele Soldaten bewogen, zu den Aufständischen überzutreten (BV II, 15 p. 482, 5; II, 26 p. 520, 10). Isaurier verrieten Rom an die Gothen (BG III, 36 p. 433, 8).

Auch die Verpflegung der Truppen wurde nicht regelmäßig wahrgenommen, so daß sich die Soldaten auf Requisitionen angewiesen sahen (BG III, 40 p. 456, 17; Agath. I, 18 p. 52, 7). Wenn solche auch einmal mit körperlicher Züchtigung bestraft wurden (BV I, 16 p. 378, 11), so konnte das nicht helfen, da die mit Weibern schwelgenden Offiziere selbst die Einwohner bedrückten und gegen die Mannschaften nachsichtig waren (BG III, 1 p. 283,$13 ; 9$ p. 312, 9), was dann 
zu Disziplinlosigkeit und Ungehorsam führte. Des Hungers wegen verrieten Isaurier Rom (BG III, 20 p. 360, 21). Konon, der Kommandant von Rom, der durch schändlichen Getreidewucher die in der Stadt herrschende Hungersnot verschlimmert hatte, wurde von den Soldaten ermordet, und da diese mit Uebertritt zu den Gothen drohten, mufite Justinian ihnen das Verbrechen verzeihen (BG III, 30 p. 401, 5).

Wie wenig wohl sich die Soldaten im Heere fühlten, zeigen die häufigen Nachrichten vom Ueberlaufen teils einzelner Leute (BG III, 11 p. 322,$22 ; 15$ p. 338,$18 ;$ IV, 31 p. 619,

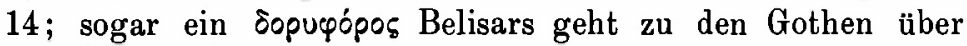
III, 35 p. 431, 2), teils größerer oder kleinerer Scharen (BG 1, 28 p. 130,3 ; III, 18 p. 354,$3 ; 26$ p. 388,3 ; letztere, 70 an der Zahl, wurden nach der Schlacht bei Taginae niedergehauen IV, 32 p. 624, 9). Anders stand es mit Kriegsgefangenen, welche, um ihr Los zu erleichtern, in das Gothenheer eintraten. So nach Uebergabe des Kastells Ruscia die Besatzungstruppen (BG III, 30 p. 404, 19) sowie die 400 Mann starke Besatzung der Moles Hadriani und 300 Mann von den Truppen in Rom (BG III, 36 p. 436, 9 und 17); ferner im Perserkriege die Besatzungen der Städte Beroea und Petra (BP II, 7 p. 186, 2; II, 17 p. 228, 17). Besonders schlimm war es, dak Herodian, der Kommandant von Spoleto, aus $\mathrm{Hak}_{3}$ gegen Belisar die Stadt den Gothen übergab und mit seinen Leuten zum Feinde übertrat (BG III, 12 p. 326, 10. Hist. arc. 5 p. 37,8$)$. Mitunter kamen römische Ueberläufer bei den Gothen zu Ehren, wie der frühere sopuyópos Belisars Gandulf (oder Indulf) dort Offizier wurde (BG IV, 23 p. 577, 11), und in Perusia zwei römische Ueberläufer, von denen der eine

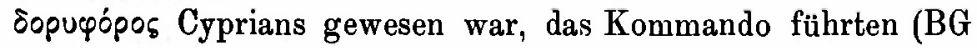
IV, 33 p. 629, 8). Was den Rücktritt von Ueberläufern anbetrifft, so hören wir, daß̧ zahlreiche Römer im Gothenheer dem Germanus in Aussicht stellen, sie würden nach seiner Ankunft in Italien zu ihm übertreten (BG III, 39 p. 448, 4), und daß Narses beabsichtigte, die Ueberläufer durch Anerbieten von Geld zu ihren alten Fahnen zurückzuführen (BG IV, 26 p. 598, 1). Näheres erfahren wir nicht.

Recht schlimm war die Disziplinlosigkeit der Truppen. 
Allerdings wird die gute Ordnung gelobt, in der das siegreiche Heer in Carthago einzog; die Leute nahmen in aller Ruhe ihre Quartierbillets in Empfang und bezahlten die Lebensmittel (BV I, 21 p. 396, 17). Aber kur' darauf bewiesen die Soldaten bei der Plünderung des vandalischen Lagers - auf Anteil an der Beute hatten sie ein Recht (BV II, 15 p. 482, 6) eine derartige Zügellosigkeit, daß, falls die Feinde einen Angriff gemacht hätten, niemand davon gekommen wäre (BV II, 4 p. 423, 22). Dasselbe wird von der Haltung der Soldaten nach der Einnahme des Lagers des Aufrührers Stotzas berichtet (BV II, 17 p. 489, 23). Besonders schwierig zeigten sich stets die barbarischen Truppen. So erklärt Belisar den Neapolitanern gegenüber, er fürchte, falls ihre Stadt erobert würde, die Wut der Barbaren nicht zügeln zu können (BG I, 9 p. 49, 5), und Narses sah sich veranlaßst, die in seinem Heere befindlichen Langobarden wegen ihrer Zügellosigkeit nach Hause zu schicken, gab ihnen jedoch Truppen mit, die sie auf dem Marsche von Ausschreitungen zurückhalten sollten (BG IV, 33 p. 627,19$)$.

Sehr auffallend ist es, dak ein vornehmer Perser sich einst gegen Chosroes äußerst anerkennend über die Haltung des Heeres unter Belisar äuBert (BP II, 21 p. 245, 12); mehr entspricht unserer Erwartung die Behauptung eines andern Persers, die Römer seien niemals in guter Ordnung in den Kampf gezogen (BP I, 14 p. 68, 5), und der Umstand, daß Belisar seine Leute zu strengem Gehorsam ermahnen muß (ibid. p. 69, 1). So kann es nicht verwundern, daf einst die Truppen, auch einige Offiziere, von Belisar in unverschämter Weise fordern, in den Kampf geführt zu werden (BP I, 18 p. 93, 4).

Ganz böse war es, wenn die höheren Offiziere sich dem Oberkommando nicht unterordnen wollten. So konnte Martinus, Magister militum Orientis, die ihm unterstellten zahlreichen Duces nicht recht zusammenhalten (BP II, 24 p. 262). Isaak unternahm gegen das Verbot Belisars eine Expedition (BG III, 19 p. 358, 18). Als Narses zum ersten Male nach Italien geschickt war, bildete er sich zu systematischer Opposition gegen Belisar eine große Partei, so daß er auf des 
letztern Bericht von Justinian zurückgerufen wurde (BG II, 18-22 p. 217 ff.). Den Oberbefehl in Italien bekam später Narses, weil Justinian, wie Procop meint, ähnliche Zustände fürchtete, wenn er den Johannes, den Neffen Vitalians, der sich große Verdienste erworben und den nächsten Anspruch auf die Führung hatte, mit demselben bekleidete. Dieser hatte zwar als Magister militum in Illyricum (BG III, 39 p. 446, 8) höheren Rang, als die in Italien amtierenden Duces, aber er war lange Zeit ihr Kollege gewesen, und daher lag die Besorgnis nahe, er werde nicht unbedingten Gehorsam finden (BG IV, 21 p. 570, 2). Uebrigens waren auch gegen Narses die Duces ungehorsam (Agath. I, 17 p. 50, 6). Eine förmliche Revolte entstand im afrikanischen Heere. Nach Besiegung der Vandalen hatten deren Witwen vielfach römische Soldaten geheiratet, diese wünschten nun in Besitz der von ihren Frauen während ihrer ersten Ehe innegehabten Ländereien zu gelangen; dieselben wurden jedoch vom Kaiser eingezogen. Außerdem waren zahlreiche Soldaten Anhänger des Arianischen Glaubens, dessen Ausübung von Justinian auf das strengste verboten war (BV II, 14 ff. p. 469 ff.). Die Geschichte dieses sehr gefährlichen Aufstandes kann hier nicht verfolgt werden.

Mit strenger Disziplin läßt es sich wohl nicht vereinigen, daß auf die Herausforderungen kampfbegieriger und renommiersüchtiger Barbaren zu Einzelkämpfen stets ohne weiteres reagiert wird. Wir bemerken darüber folgendes. BP I, 13 p. $64 \mathrm{f}$. wird von einem jüngeren und einem älteren Perser erzählt, auf deren Herausforderung sich beide Male ein gewisser Andreas meldet, der kein Soldat war, sich aber als Turnlehrer in einer Palästra zu Byzanz große Gewandtheit erworben hatte und zur persönlichen Pflege des Buzes, des Kommandanten am Libanon, mit in den Krieg gezogen war. In beiden Fällen trug er den Sieg davon. BG III, 4 p. 296, 20 ist der Fordernde ein Gothe; er sowohl wie sein Gegner, ein Armenier, gehen zu Grunde. BG IV, 31 p. 619, 10 fordert ein römischcr Ueberläufer; ihn besiegt ein Eopupópos des Narses. Anders liegt die Sache in folgenden Fällen. Im Vandalenkriege (BV II, 13 p. 464, 6) wïnschte der Anführer der Mauren für seine Leute die Benutzung einer von den Römern besetzten Quelle 
zu erreichen und versprach dafür einen Teil der Beute. Der Anführer der Römer lehnte jedoch dieses Anerbieten ab und machte die Gewährung des Gesuches von dem Ausfall eines Zweikampfes zwischen den beiden Fubrern abhängig, in dem er Sieger blieb. Persönliche Feindschaft führte den Rebellenführer Stotzas mit Johannes, dem Sohne des Sisinniolus, im Kampfe zusammen; beide Offiziere fanden den Tod (BV 24 p. 514,6$)$.

In betreff der Strafen ist folgendes hervorzuheben. Während in älterer Kaiserzeit Verhaftung nur zur Brechung des Ungehorsams sowie als Untersuchungs- und Exekutionshaft vorkam, bestrafte Justinian den Dagisthaeus, der im Lande der Lazen kommandiert hatte und von diesen wegen persischer Gesinnung verklagt war, mit Gefängnis (BG IV, 9 p. 497, 19), befreite ihn aber nach etwa Jahresfrist, um ihn mit Narses nach Italien zu schicken (BG IV, 26 p. 598, 23). Die Strafe des Verbrennens, die früher für Verrat vorgekommen war (Ammian. Marc. 29, 4, 7), wird einmal erwähnt. Ein römischer Soldat hatte sich verleiten lassen, Briefe der in Auximum belagerten Gothen an Vitigis zu überbringen; als das bekannt geworden war, überlief ihn Belisar seinen Kameraden zur Bestrafung, die ihn dann verbrannten (BG II, 26 p. 255, 9). Körperliche Züchtigung ist bereits S. 133 erwähnt.

Auch über die barbarischen Hilfsvölker nahmen die Oberfeldherren die Strafgewalt in Anspruch. Als zwei Massageten in der Trunkenheit einen Kameraden erschlagen hatten, ließ3 Belisar sie aufhängen. Dagegen machten die Massageten geltend, sie seien nur Bundesgenossen und brauchten sich nicht wach römischen Gesetzen richten zu lassen; bei ihnen sei für Totschläge solche Strafe nicht üblich. Auch römische Soldaten tadelten Belisar, der durch eine Ansprache über die Notwendigkeit guter Zucht und strenger Nüchternheit seine Leute beruhigte (BV I, 12 p. 364, 2).

Narses zog einen vornehmen Heruler, der einen seiner Diener eines Versehens wegen getötet hatte, zur Verantwortung; als derselbe das große Wort hatte und auf sein Recht pochte, lief ihn der Feldherr durch einen sopuyópos töten. Zunächst wollten die Heruler sich nicht weiter am Kampfe 
beteiligen, zuletzt beruhigten sie sich und traten in guter Ordnung an (Agath. II, 7 p. 78, 12).

Von Belohnungen ist wenig die Rede. Wie Narses die Tapferkeit eines Katalogsoldaten belohnte, ist bereits oben S. 118 gesagt, sowie S. 119, daß Belisar Armbänder und Halsketten verschenkte. $B G I V, 31$ p. 619,5 läßt Narses vor der Schlacht Armbänder, Halsketten und goldgeschmïckte Zügel auf Stangen den Leuten zeigen, um ihren Mut zu beleben. Man wolle jedoch nicht annehmen, daß̉ damit die früher übliche Verleihung von dona militaria wieder eingeführt worden sei.

Nach den vorstehenden Ausführungen waren die Zustände im Heere Justinians sehr traurig. Die aus den Provinzen des weiten Reiches sich rekrutierenden Truppen entbehrten nicht nur der nationalen Einheit und damit der rechten Hingabe an Kaiser und Reich, sondern auch des echten militärischen Geistes und der Kriegstüchtigkeit. Die überaus zahlreichen und kräftigeren Barbaren dienten selbstverständlich nur in der Aussicht auf einen reichen Gewinn. Auch die Generale waren nicht weniger darauf bedacht, Reichtamer zu erwerben. Wenn unter diesen Umständen die Armee doch noch Tüchtiges leistete und zwei Provinzen wieder erwarb, so ist dies einerseits den Verhältnissen der Feinde zuzuschreiben, ' von denen die Vandalen durch den hundertjährigen Aufenthalt in dem üppigen Afrika verweichlicht waren, die Gothen sich nicht in der Lage befanden, die im Kriege entstandenen Lücken auszufüllen, andrerseits der überlegenen Kriegskunst der Römer und besonders der groken Tüchtigkeit der Oberbefehlshaber, deren Namen daher noch jetzt mit großer Ehre genannt werden. Hannover. Albert Müller. 